\title{
Slip Effects on the Unsteady MHD Pulsatile Blood Flow through Porous Medium in an Artery under the Effect of Body Acceleration
}

\author{
Islam M. Eldesoky \\ Basic Engineering Sciences Department, Faculty of Engineering, Menoufia University, Egypt \\ Correspondence should be addressed to Islam M. Eldesoky, eldesokyi@yahoo.com
}

Received 30 March 2012; Accepted 28 June 2012

Academic Editor: R. H. J. Grimshaw

Copyright (C) 2012 Islam M. Eldesoky. This is an open access article distributed under the Creative Commons Attribution License, which permits unrestricted use, distribution, and reproduction in any medium, provided the original work is properly cited.

\begin{abstract}
Unsteady pulsatile flow of blood through porous medium in an artery has been studied under the influence of periodic body acceleration and slip condition in the presence of magnetic field considering blood as an incompressible electrically conducting fluid. An analytical solution of the equation of motion is obtained by applying the Laplace transform. With a view to illustrating the applicability of the mathematical model developed here, the analytic explicit expressions of axial velocity, wall shear stress, and fluid acceleration are given. The slip condition plays an important role in shear skin, spurt, and hysteresis effects. The fluids that exhibit boundary slip have important technological applications such as in polishing valves of artificial heart and internal cavities. The effects of slip condition, magnetic field, porous medium, and body acceleration have been discussed. The obtained results, for different values of parameters into the problem under consideration, show that the flow is appreciably influenced by the presence of Knudsen number of slip condition, permeability parameter of porous medium, Hartmann number of magnetic field, and frequency of periodic body acceleration. The study is useful for evaluating the role of porosity and slip condition when the body is subjected to magnetic resonance imaging (MRI).
\end{abstract}

\section{Introduction}

The investigations of blood flow through arteries are of considerable importance in many cardiovascular diseases particularly atherosclerosis. The pulsatile flow of blood through an artery has drawn the attention of researchers for a long time due to its great importance in medical sciences. Under normal conditions, blood flow in the human circulatory system depends upon the pumping action of the heart and this produces a pressure gradient throughout the arterial network. Chaturani and Palanisamy [1] studied pulsatile flow of 
blood through a rigid tube under the influence of body acceleration as a Newtonian fluid. Elsoud et al. [2] studied the interaction of peristaltic flow with pulsatile couple stress fluid. The mathematical model considers a viscous incompressible couple stress fluid between infinite parallel walls on which a sinusoidal travelling wave is imposed. El-Shehawey et al. [3] investigated the pulsatile flow of blood through a porous medium under periodic body acceleration. The arterial MHD pulsatile flow of blood under periodic body acceleration has been studied by Das and Saha [4]. Assuming blood to be an incompressible biviscous fluid, the effect of uniform transverse magnetic field on its pulsatile motion through an axisymmetric tube was analyzed by Sanyal and Biswas [5]. Rao et al. [6] analyzed the flow of combined two phase motion of viscous ideal medium through a parallel plate channel under the influence of an imposed pressure gradient and periodic body acceleration.

During recent years, the effect of magnetic field on the flow of viscous fluid through a uniform porous media has been the subject of numerous applications. The red blood cell (RBC) is a major biomagnetic substance, and the blood flow may be influenced by the magnetic field. In general, biological systems are affected by an application of external magnetic field on blood flow, through human arterial system. The presence of the stationary magnetic field contributes to an increase in the friction of flowing blood. This is because the anisotropic orientation of the red blood cells in the stationary magnetic field disturbs the rolling of the cells in the flowing blood and thereby the viscosity of blood increases. The properties of human blood as well as blood vessels and magnetic field effect were the subjects of interest for several researchers. Mekheimer [7] investigated the effect of a magnetic field on peristaltic transport of blood in a non-uniform two-dimensional channel. The blood is represented by a viscous, incompressible, and electrically conducting couple stress fluid. A mathematical model for blood flow in magnetic field is studied by Tzirtzilakis [8]. This model is consistent with the principles of ferrohydrodynamics and magnetohydrodynamics and takes into account both magnetization and electrical conductivity of blood. Jain et al. [9] investigated a mathematical model for blood flow in very narrow capillaries under the effect of transverse magnetic field. It is assumed that there is a lubricating layer between red blood cells and tube wall. Fluid flow analysis of blood flow through multistenosis arteries in the presence of magnetic field is investigated by Verma and Parihar [10]. In this investigation, the effect of magnetic field and shape of stenosis on the flow rate is studied. Singh and Rathee [11] studied the analytical solution of two-dimensional model of blood flow with variable viscosity through an indented artery due to LDL effect in the presence of magnetic field.

Porous medium is defined as a material volume consisting of solid matrix with an interconnected void. It is mainly characterized by its porosity, ratio of the void space to the total volume of the medium. Earlier studies in flow in porous media have revealed the Darcy law which relates linearly the flow velocity to the pressure gradient across the porous medium. The porous medium is also characterized by its permeability which is a measure of the flow conductivity in the porous medium. An important characteristic for the combination of the fluid and the porous medium is the tortuosity which represents the hindrance to flow diffusion imposed by local boundaries or local viscosity. The tortuosity is especially important as related to medical applications [12]. Flow through porous medium has been studied by a number of workers employing Darcy's law. A mathematical modeling of blood flow in porous vessel having double stenosis in the presence of an external magnetic field has been investigated by Sinha et al. [13]. The magnetohydrodynamics effects on blood flow through a porous channel have been studied by Ramamurthy and Shanker [14]. Eldesoky and Mousa [15] investigated the peristaltic flow of a compressible non-Newtonian Maxwellian fluid through porous medium in a tube. Reddy and Venkataramana [16] investigated the 
peristaltic transport of a conducting fluid through a porous medium in an asymmetric vertical channel.

No slip boundary conditions are a convenient idealization of the behavior of viscous fluids near walls. The inadequacy of the no-slip condition is quite evident in polymer melts which often exhibit microscopic wall slip. The slip condition plays an important role in shear skin, spurt, and hysteresis effects. The boundary conditions relevant to flowing fluids are very important in predicting fluid flows in many applications. The fluids that exhibit boundary slip have important technological applications such as in polishing valves of artificial heart and internal cavities [17]. The slip effects on the peristaltic flow of a nonNewtonian Maxwellian fluid have been investigated by El-Shehawy et al. [18]. The influence of slip condition on peristaltic transport of a compressible Maxwell fluid through porous medium in a tube has been studied by Eldesoky [19]. Many recent researches have been made in the subject of slip boundary conditions [20-25].

In situations like travel in vehicles, aircraft, operating jackhammer, and sudden movements of body during sports activities, the human body experiences external body acceleration. Prolonged exposure of a healthy human body to external acceleration may cause serious health problem like headache, increase in pulse rate and loss of vision on account of disturbances in blood flow [6]. Many mathematical models have already been investigated by several research workers to explore the nature of blood flow under the influence of external acceleration. Sometimes human being suffering from cardiogenic or anoxic shock may deliberately be subjected to whole body acceleration as a therapeutic measure [4]. El-Shahed [26] studied pulsatile flow of blood through a stenosed porous medium under periodic body acceleration. El-Shehawey et al. [3, 27-30] studied the effect of body acceleration in different situations. They studied the effect of MHD flow of blood under body acceleration. Also, studied Womersley problem for pulsatile flow of blood through a porous medium. The flow of MHD of an elastic-viscous fluid under periodic body acceleration has been studied. The blood flow through porous medium under periodic body acceleration has been studied.

In the present paper, the effect of slip condition on unsteady blood flow through a porous medium has been studied under the influence of periodic body acceleration and an external magnetic field. The analysis is carried out by employing appropriate analytical methods and some important predictions have been made basing upon the study. This investigation can play a vital role in the determination of axial velocity, shear stress, and fluid acceleration in particular situations. Since this study has been carried out for a situation when the human body is subjected to an external magnetic field, it bears the promise of significant application in magnetic or electromagnetic therapy, which has gained enough popularity. The study is also useful for evaluating the role of porosity and slip condition when the body is subjected to magnetic resonance imaging (MRI).

\section{Mathematical Modeling of the Problem}

Consider the unsteady pulsatile flow of blood in an axisymmetric cylindrical artery of radius $R$ through porous medium with body acceleration. The fluid subjected to a constant magnetic field acts perpendicular to the artery as in Figure 1. Induced magnetic field and external electric field are neglected. The slip boundary conditions are also taken into account. The cylindrical coordinate system $(r, \theta, z)$ are introduced with $z$-axis lies along the center of the 


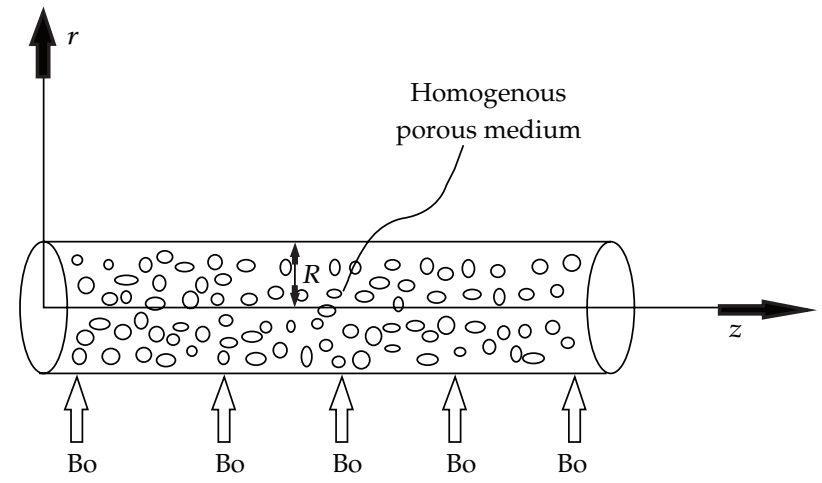

Figure 1: schematic diagram for the flow geometry.

artery and $r$ transverse to it. The pressure gradient and body acceleration are respectively given by

$$
\begin{gathered}
-\frac{\partial p}{\partial z}=A_{o}+A_{1} \cos \left(\omega_{p} t\right), \\
G=a_{o} \cos \left(\omega_{b} t\right),
\end{gathered}
$$

where $A_{o}$ and $A_{1}$ are pressure gradient of steady flow and amplitude of oscillatory part respectively, $a_{o}$ is the amplitude of the body acceleration, $\omega_{p}=2 \pi f_{p}, \omega_{b}=2 \pi f_{b}$ with $f_{p}$ is the pulse frequency, and $f_{b}$ is the body acceleration frequency and $t$ is time. by

The governing equation of the motion for flow in cylindrical polar coordinates is given

$$
\rho \frac{\partial u}{\partial t}=-\frac{\partial p}{\partial z}+\mu \nabla^{2} u+\rho G-\left(\frac{\mu}{k}\right) u+\bar{J} \times \bar{B}
$$

Maxwell's equations are

$$
\bar{\nabla} \cdot \bar{B}=0, \quad \bar{\nabla} \times \bar{B}=\mu_{o} \bar{J}, \quad \bar{\nabla} \times \bar{E}=-\frac{\partial \bar{B}}{\partial t} .
$$

Ohm's law is

$$
\bar{J}=\sigma(\bar{E}+\bar{V} \times \bar{B})
$$

where $\bar{V}=(0,0, u)$ is the velocity distribution, $\rho$ the blood density, $\mu_{0}$ magnetic permeability, $\bar{B}=\left(0, B_{o}, 0\right)$ the magnetic field, $\bar{E}$ the electric field, $\bar{J}$ the current density, $k$ is the permeability parameter of porous medium, $\mu$ the dynamic viscosity of the blood, and $\sigma$ the 
electric conductivity of the blood. For small magnetic Reynolds number, the linearlized magnetohydrodynamic force $\bar{J} \times \bar{B}$ can be put into the following form:

$$
\bar{J} \times \bar{B}=-\sigma B_{O}^{2} u
$$

where $u(r, t)$ represents the axial velocity of the blood.

The shear stress $\tau$ is given by [13] as

$$
\tau=-\mu \frac{\partial u}{\partial r}
$$

Under the above assumptions the equation of motion is

$$
\rho \frac{\partial u}{\partial t}=A_{o}+A_{1} \cos \left(\omega_{p} t\right)+\mu\left(\frac{\partial^{2} u}{\partial r^{2}}+\frac{1}{r} \frac{\partial u}{\partial r}\right)+\rho\left(a_{o} \cos \left(\omega_{b} t\right)\right)-\left(\frac{\mu}{k}\right) u-\sigma B_{O}^{2} u
$$

The boundary conditions that must be satisfied by the blood on the wall of artery are the slip conditions. For slip flow the blood still obeys the Navier-Stokes equation, but the no-slip condition is replaced by the slip condition $u_{t}=A_{p} \partial u_{t} / \partial n$, where $u_{t}$ is the tangential velocity, $n$ is normal to the surface, and $A_{p}$ is a coefficient close to the mean free path of the molecules of the blood [31]. Although the Navier condition looked simple, analytically it is much more difficult than the no-slip condition, and then the boundary conditions on the wall of the artery are

$$
\begin{gathered}
u(0, t) \text { is finite at } r=0, \\
u(R, t)=\left.A_{p} \frac{\partial u(r, t)}{\partial r}\right|_{r=R^{\prime}},(\text { Slip condition }) .
\end{gathered}
$$

Let us introduce the following dimensionless quantities:

$$
\begin{gathered}
u^{*}=\frac{u}{\omega R}, \quad r^{*}=\frac{r}{R}, \quad t^{*}=t \omega, \quad A_{o}^{*}=\frac{R}{\mu \omega} A_{o}, \\
A_{1}^{*}=\frac{R}{\mu \omega} A_{1}, \quad a_{o}^{*}=\frac{\rho R}{\mu \omega} a_{o}, \quad z^{*}=\frac{z}{R^{\prime}}, \quad k^{*}=\frac{k}{R^{2}}, \quad b=\frac{\omega_{b}}{\omega_{p}} .
\end{gathered}
$$

The Hartmann number Ha, the Womersley parameter $\alpha$, and the Knudsen number kn, are defined respectively by

$$
\mathrm{Ha}=B_{0} R \sqrt{\frac{\sigma}{\mu}}, \quad \alpha=R \sqrt{\frac{\rho \omega}{\mu}}, \quad \mathrm{kn}=\frac{A}{R} .
$$


Under the above assumptions (2.7) and (2.8) can be rewritten in the non-dimensional form after dropping the stars as

$$
\alpha^{2} \frac{\partial u}{\partial t}=A_{o}+A_{1} \cos (t)+a_{o} \cos (b t)+\frac{\partial^{2} u}{\partial r^{2}}+\frac{1}{r} \frac{\partial u}{\partial r}-\left(H a^{2}+\frac{1}{k}\right) u
$$

Also the boundary conditions are

$$
\begin{aligned}
& u(0, t) \text { is finite at } r=0 \\
& u(1, t)=\left.\mathrm{kn} \frac{\partial u(r, t)}{\partial r}\right|_{r=1} .
\end{aligned}
$$

And the initial condition is

$$
u(r, 0)=1 \quad(\text { at } t=0)
$$

\section{Solution of the Problem}

Applying Laplace Transform to (2.11), we get

$$
\begin{aligned}
\alpha^{2}\left(s u^{*}(r, s)-u^{*}(r, o)\right)= & A_{o}\left(\frac{1}{s}\right)+A_{1}\left(\frac{s}{s^{2}+1}\right)+a_{o}\left(\frac{s}{s^{2}+b^{2}}\right) \\
& +\frac{d^{2} u^{*}}{d r^{2}}+\frac{1}{r} \frac{d u^{*}}{d r}-\left(\mathrm{Ha}^{2}+\frac{1}{k}\right) u^{*}
\end{aligned}
$$

where $u^{*}(r, s)=\int_{0}^{\infty} u(r, t) e^{-s t} d t,(s>0)$.

Substituting by the I.C. equation (2.12c) into (3.1) and dropping the stars, we get

$$
r^{2} \frac{d^{2} u}{d r^{2}}+r \frac{d u}{d r}-\lambda^{2} r^{2} u=-r^{2} G
$$

where

$$
\begin{gathered}
\lambda^{2}=\alpha^{2} s+\mathrm{Ha}^{2}+\frac{1}{k}=\alpha^{2}\left(s+\frac{\mathrm{Ha}^{2}+(1 / k)}{\alpha^{2}}\right), \\
G=\alpha^{2}+A_{o}\left(\frac{1}{s}\right)+A_{1}\left(\frac{s}{s^{2}+1}\right)+a_{o}\left(\frac{s}{s^{2}+b^{2}}\right) .
\end{gathered}
$$

Homogenous solution is as follows:

$$
r^{2} \frac{d^{2} u}{d r^{2}}+r \frac{d u}{d r}-\lambda^{2} r^{2} u=0
$$


This equation is modified Bessel differential equation so the solution is

$$
u_{h}=C_{1} I_{O}(\lambda r)+C_{2} K_{O}(\lambda r)
$$

where $I_{O}$ and $K_{O}$ are modified Bessel functions of order zero. Since the solution is bounded at $r=0$, then the constant $C_{2}$ equals zero, then

$$
u_{h}=C_{1} I_{O}(\lambda r)
$$

We can get the particular solution using the undetermined coefficients as the following:

$$
\begin{gathered}
u_{p}=\beta_{1}+\beta_{2} r, \\
\frac{d u_{p}}{d r}=\beta_{2}, \quad \frac{d^{2} u_{p}}{d r^{2}}=0 .
\end{gathered}
$$

Substituting into (3.2) and comparing the coefficients of $r$ and $r^{2}$ we get

$$
u_{p}=\frac{G}{\lambda^{2}}
$$

The general solution is

$$
u_{g}=u_{h}+u_{p}=C_{1} I_{O}(\lambda r)+\frac{G}{\lambda^{2}} .
$$

Substituting from (2.12b) into (3.9) to calculate the constant $C_{1}$ we get

$$
C_{1}=\frac{-\left(G / \lambda^{2}\right)}{-\operatorname{kn} \lambda I_{1}(\lambda)+I_{o}(\lambda)}
$$

Then the general solution can obtained on the following form:

$$
u_{g}(r, s)=\frac{G}{\lambda^{2}}\left(1-\frac{I_{O}(\lambda r)}{I_{O}(\lambda)-\mathrm{kn} \lambda I_{1}(\lambda)}\right) .
$$

For the sake of analysis, the part $\left(1-\left(\left(I_{O}(\lambda r)\right) /\left(I_{O}(\lambda)-\operatorname{kn} \lambda I_{1}(\lambda)\right)\right)\right)$ which represents an infinite convergent series as its limit tends to zero when $r$ tends to one and kn tends to zero has been approximated $[32,33]$. 
The final form of the general solution as a function of $r$ and $s$ is

$$
\begin{aligned}
u_{g}(r, s) \\
=16\left(1-r^{2}-2 k n\right) \\
\quad \times\left(\frac{\alpha^{2}+A_{o}(1 / s)+A_{1}\left(s /\left(s^{2}+1\right)\right)+a_{o}\left(s /\left(s^{2}+b^{2}\right)\right)}{64+16\left(\alpha^{2} s+\left(\left(\mathrm{Ha}^{2}+(1 / k) / \alpha^{2}\right)\right)\right)(1-2 \mathrm{kn})+\left(\alpha^{2}\left(s+\left(\left(\mathrm{Ha}^{2}+(1 / k)\right) / \alpha^{2}\right)\right)\right)^{2}(1-4 \mathrm{kn})}\right) \\
\quad+\left(1-r^{4}-4 \mathrm{kn}\right) \\
\quad \times\left(\frac{\left(\alpha^{2}\left(s+\left(\left(\mathrm{Ha}^{2}+(1 / k)\right) / \alpha^{2}\right)\right)\right)\left(\alpha^{2}+A_{o}(1 / s)+A_{1}\left(s /\left(s^{2}+1\right)\right)+a_{o}\left(s /\left(s^{2}+b^{2}\right)\right)\right)}{64+16\left(\alpha^{2}\left(s+\left(\left(\mathrm{Ha}^{2}+(1 / k)\right) / \alpha^{2}\right)\right)\right)(1-2 \mathrm{kn})+\left(\alpha^{2}\left(s+\left(\left(\mathrm{Ha}^{2}+(1 / k)\right) / \alpha^{2}\right)\right)\right)^{2}(1-4 \mathrm{kn})}\right) .
\end{aligned}
$$

Rearranging the terms and taking the inversion of Laplace Transform of (3.12) which gives the final solution as

$$
\begin{aligned}
u_{g}(r, t)= & 16\left(1-r^{2}-2 \mathrm{kn}\right)\left\{(-1 / 16)\left(M_{0}\right)+A_{o} k^{2}\left(M_{1}\right)+A_{1} k^{2}\left(M_{2}\right)+a_{o} k^{2}\left(M_{3}\right)\right\} \\
& +\left(1-r^{4}-4 \mathrm{kn}\right)\left\{\alpha^{2} k\left(M_{4}\right)+A_{o} k\left(M_{5}\right)+A_{1} k\left(M_{6}\right)+a_{o} k\left(M_{7}\right)\right\} .
\end{aligned}
$$

The expression for the shear stress is given by

$$
\begin{aligned}
\tau(r, t)= & \mu 16(2 r)\left\{(-1 / 16)\left(M_{0}\right)+A_{o} k^{2}\left(M_{1}\right)+A_{1} k^{2}\left(M_{2}\right)+a_{o} k^{2}\left(M_{3}\right)\right\} \\
& +\mu\left(4 r^{3}\right)\left\{\alpha^{2} k\left(M_{4}\right)+A_{o} k\left(M_{5}\right)+A_{1} k\left(M_{6}\right)+a_{o} k\left(M_{7}\right)\right\} .
\end{aligned}
$$

The expression for the fluid acceleration is given by:

$$
F(r, t)=\frac{\partial u}{\partial t}
$$

\section{Numerical Results and Discussion}

We studied unsteady pulsatile flow of blood through porous medium in an artery under the influence of periodic body acceleration and slip condition in the presence of magnetic field considering blood as an incompressible electrically conducting fluid. The artery is considered a circular tube. We have shown the relation between the different parameters of motion such as Hartmann number Ha, Knudsen number $\mathrm{kn}$, Womersley parameter $\alpha$, frequency of the body acceleration $b$, the permeability parameter of porous medium $k$, and the axial velocity, shear stress, fluid acceleration to investigate the effect of changing these parameters on the flow of the fluid. Hence, we can be controlling the process of flow. 


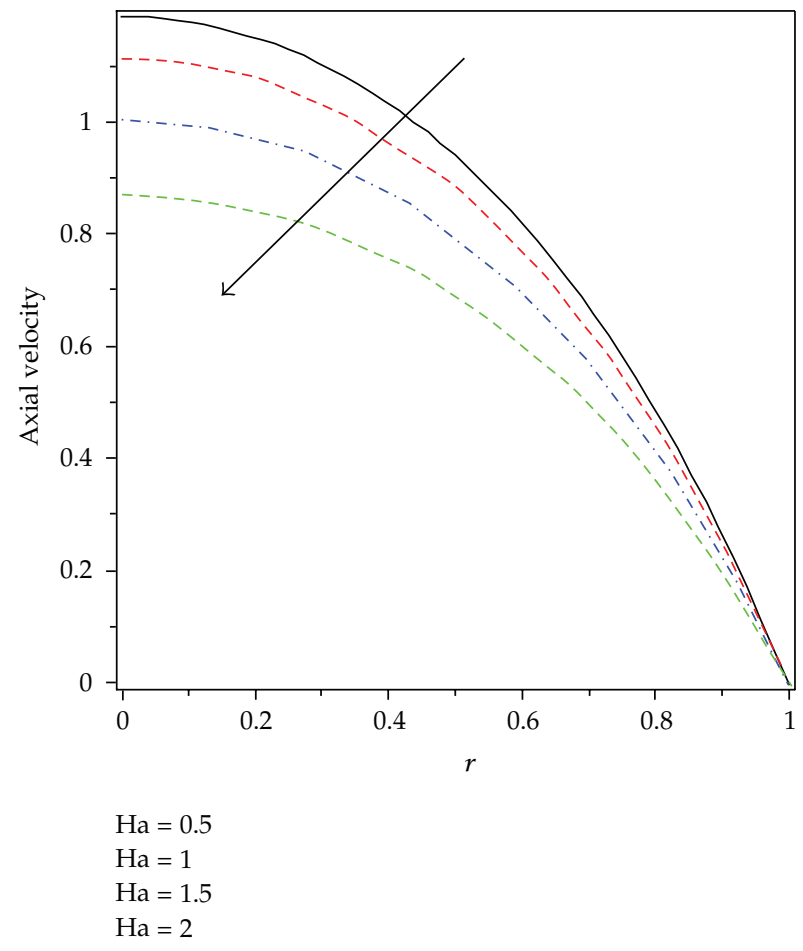

Figure 2: Effect of Hartmann number on the axial velocity $b=2, \alpha=3, a_{o}=3, A_{o}=2, A_{1}=4, t=1$, $\mathrm{kn}=$ 0.001 , and $k=0.5$.

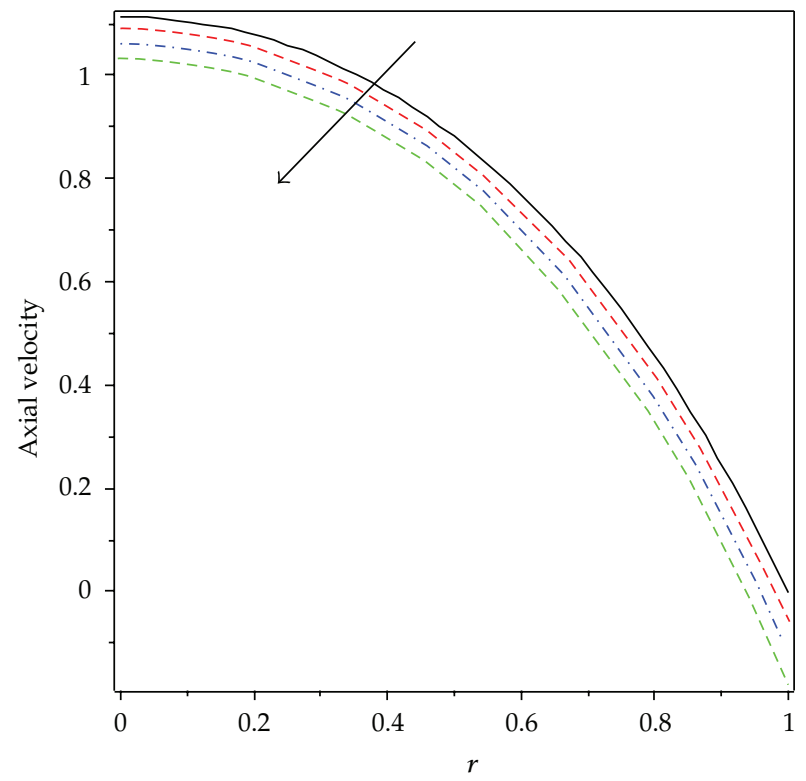

$$
\begin{aligned}
& \mathrm{kn}=0.001 \\
& \mathrm{kn}=0.02 \\
& \mathrm{kn}=0.04 \\
& \mathrm{kn}=0.06
\end{aligned}
$$

Figure 3: Effect of Knudsen number on the axial velocity $b=2, \alpha=3, a_{o}=3, A_{o}=2, A_{1}=4, t=1, \mathrm{Ha}=$ 1.0 , and $k=0.5$. 


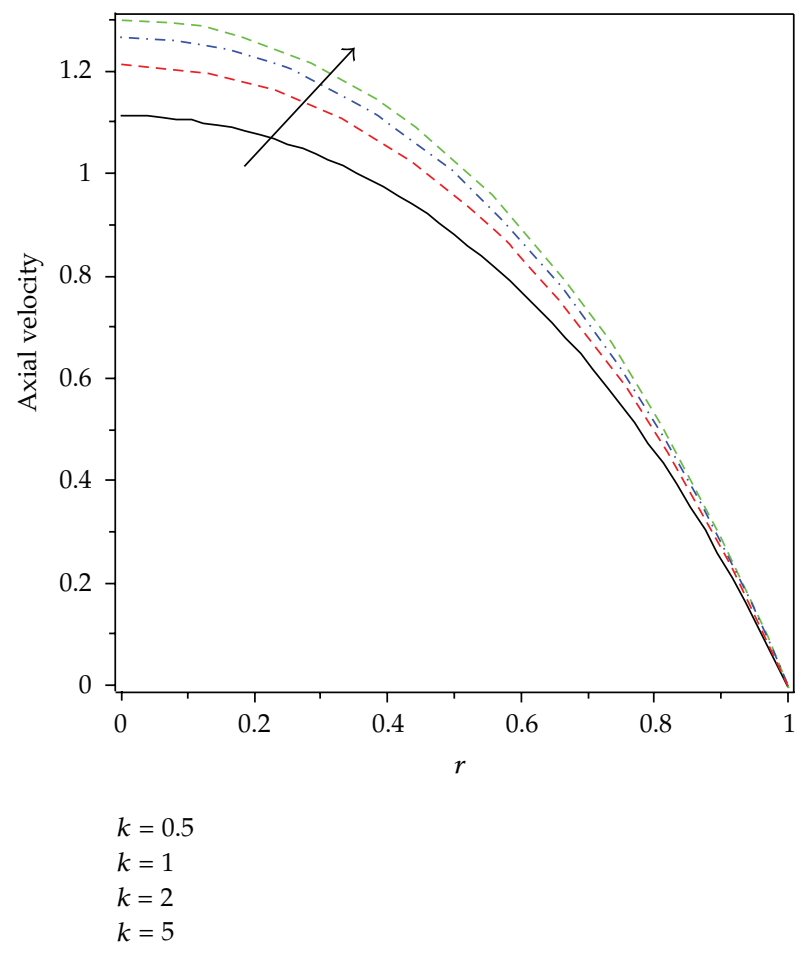

Figure 4: Effect of permeability parameter on the axial velocity $b=2, \alpha=3, a_{o}=3, A_{o}=2, A_{1}=4, t=$ $1, \mathrm{kn}=0.001$, and $\mathrm{Ha}=1.0$.

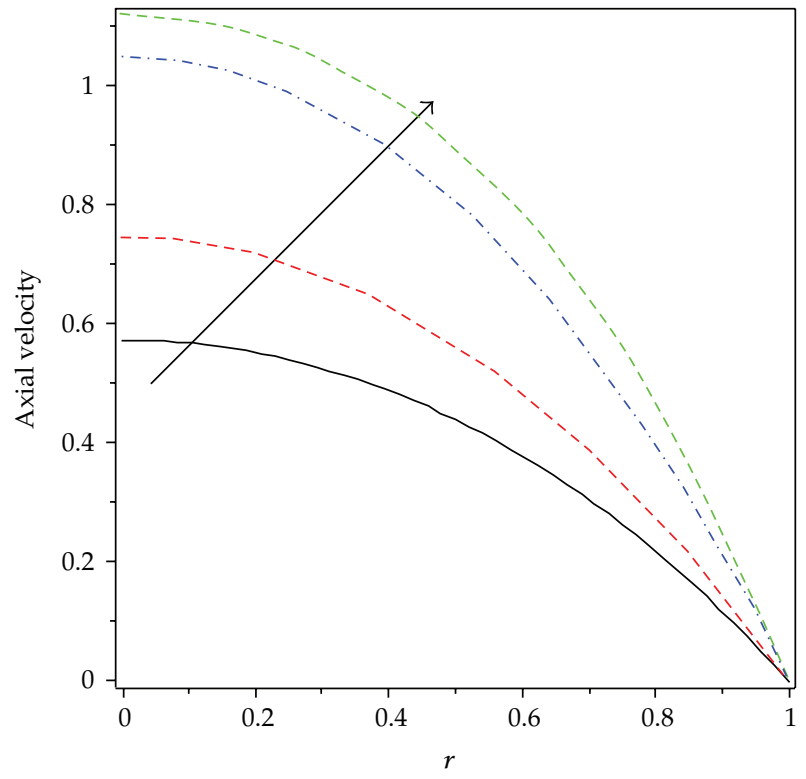

$$
\begin{aligned}
& \alpha=1 \\
& \alpha=3 \\
& \alpha=5 \\
& \alpha=7
\end{aligned}
$$

Figure 5: Effect of Womersley parameter on the axial velocity $b=2$, Ha $=1, a_{o}=3, A_{o}=2, A_{1}=4, t=$ $1, \mathrm{kn}=0.001$, and $k=0.5$. 


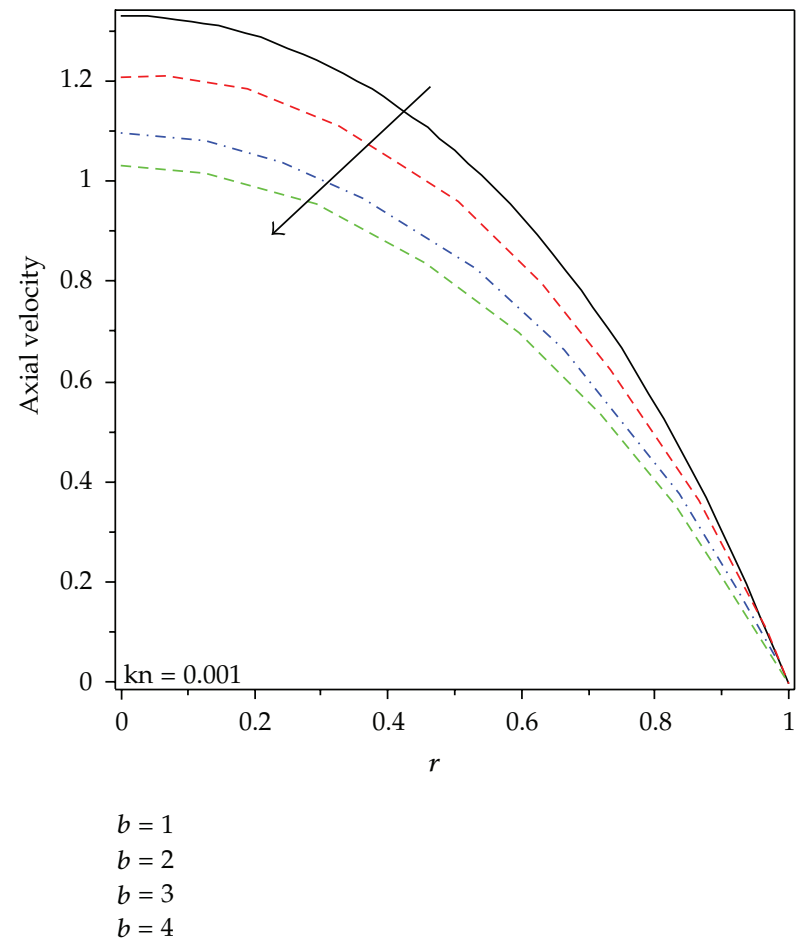

Figure 6: Effect of frequency of body acceleration on the axial velocity at $\mathrm{kn}=0.001, \alpha=3, \mathrm{Ha}=1, a_{o}=$ 3, $A_{o}=2, A_{1}=4, t=1, \mathrm{kn}=0.001$, and $k=0.5$.

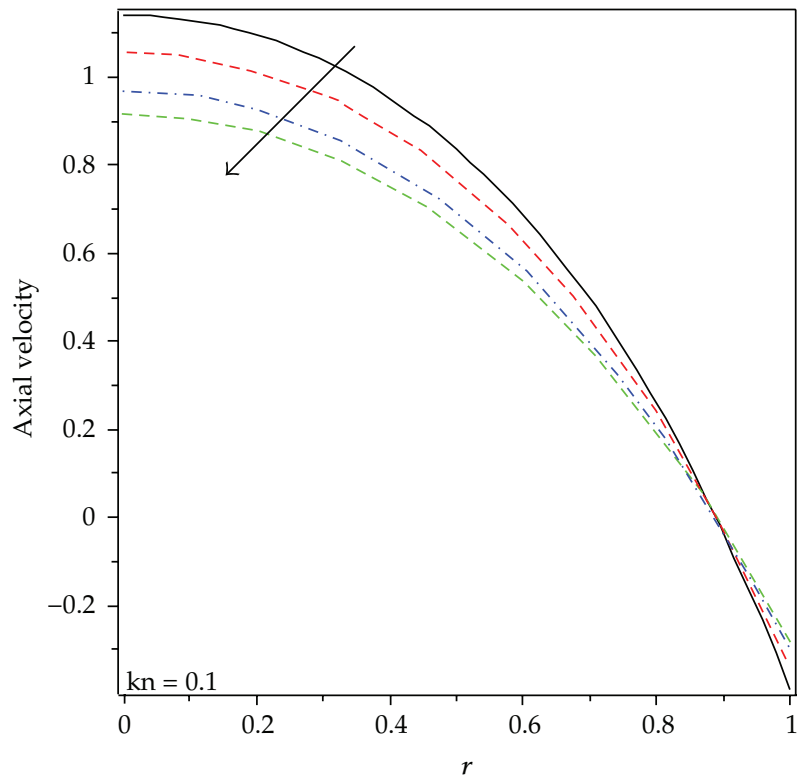

$$
\begin{aligned}
& b=1 \\
& b=2 \\
& b=3 \\
& b=4
\end{aligned}
$$

Figure 7: Effect of frequency of body acceleration on the axial velocity at $\mathrm{kn}=0.1, \alpha=3, \mathrm{Ha}=1, a_{o}=$ 3, $A_{o}=2, A_{1}=4, t=1, \mathrm{kn}=0.1$, and $k=0.5$. 


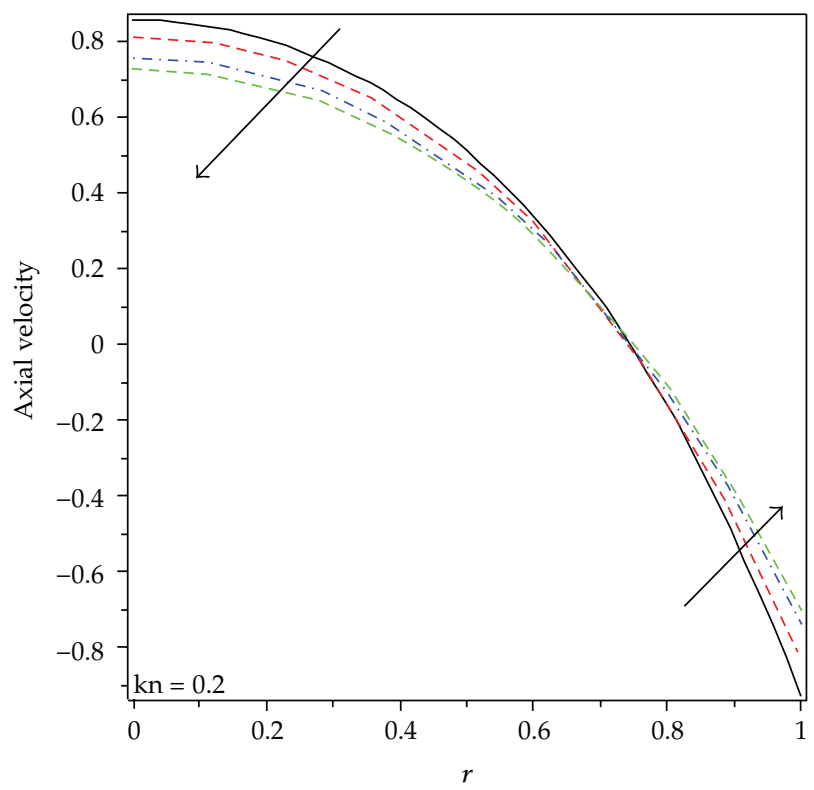

$$
\begin{aligned}
& b=1 \\
& b=2 \\
& b=3 \\
& b=4
\end{aligned}
$$

Figure 8: Effect of frequency of body acceleration on the axial velocity at $\mathrm{kn}=0.2, \alpha=3, \mathrm{Ha}=1, a_{o}=$ 3, $A_{o}=2, A_{1}=4, t=1, \mathrm{kn}=0.2$, and $k=0.5$.

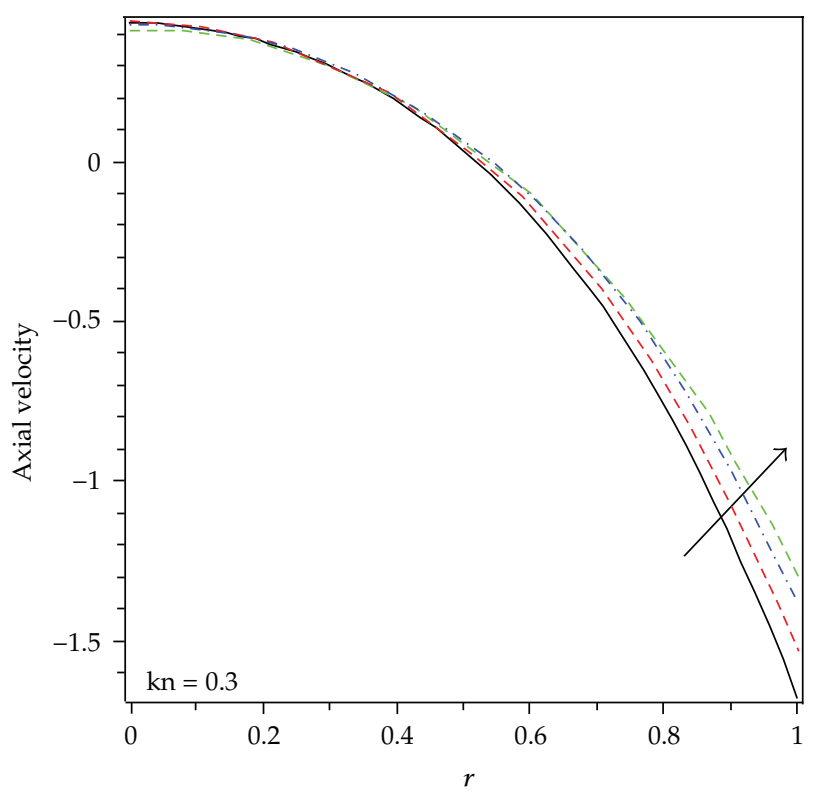

$$
\begin{aligned}
& b=1 \\
& b=2 \\
& b=3 \\
& b=4
\end{aligned}
$$

Figure 9: Effect of frequency of body acceleration on the axial velocity at $\mathrm{kn}=0.3, \alpha=3$, Ha $=1, a_{o}=$ $3, A_{o}=2, A_{1}=4, t=1, \mathrm{kn}=0.3$, and $k=0.5$. 


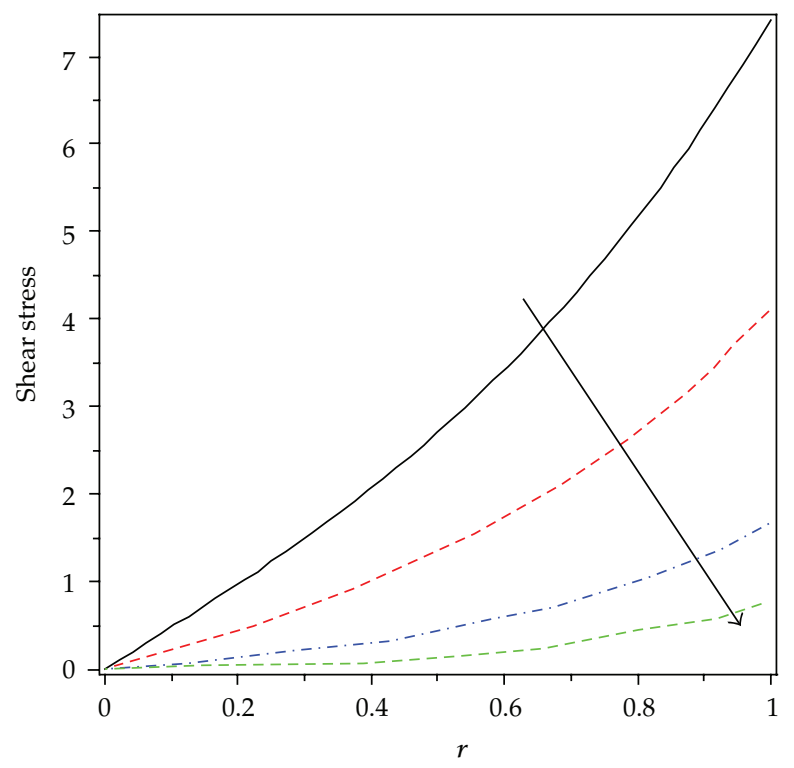

$$
\begin{aligned}
& \mathrm{Ha}=1 \\
& \mathrm{Ha}=3 \\
& \mathrm{Ha}=5 \\
& \mathrm{Ha}=7
\end{aligned}
$$

Figure 10: Effect of Hartmann number on the shear stress $\alpha=3, b=2, a_{o}=3, A_{o}=2, A_{1}=4, t=1$, $\mathrm{kn}=$ 0.01 , and $k=0.5$.

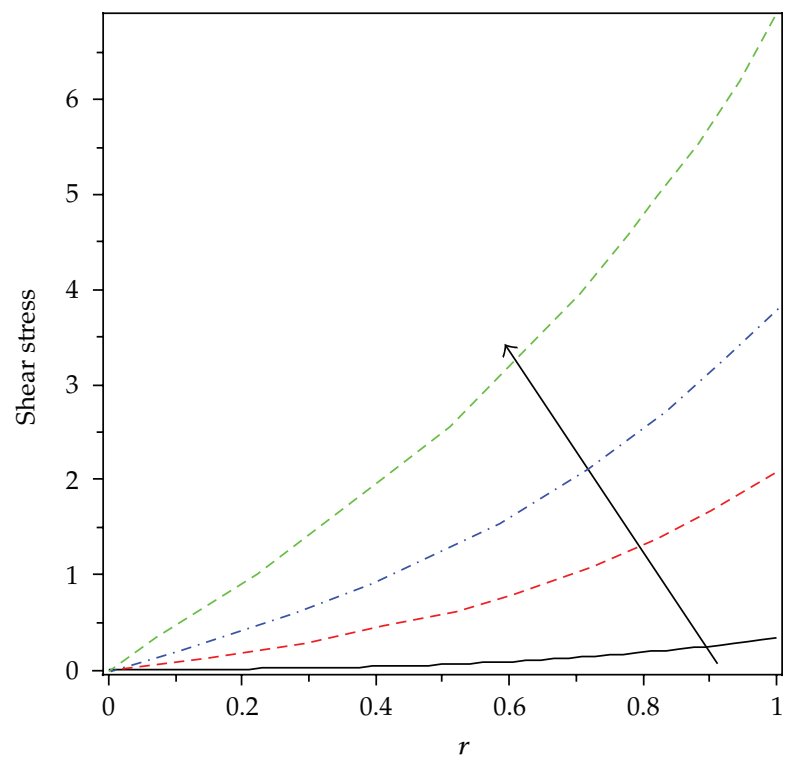

$$
\begin{aligned}
& k=0.01 \\
& k=0.05 \\
& k=0.1 \\
& k=0.5
\end{aligned}
$$

Figure 11: Effect of permeability parameter on the shear stress $\alpha=3$, Ha $=1, a_{o}=3, A_{o}=2, A_{1}=4, t=1$, $\mathrm{kn}=0.01$, and $b=2$. 


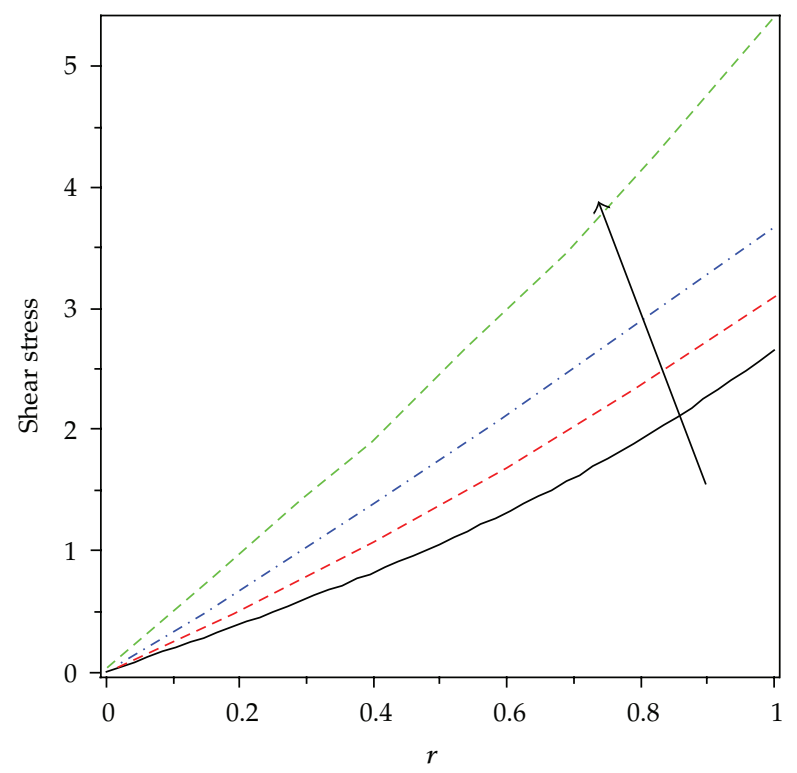

$$
\begin{aligned}
& \alpha=1 \\
& \alpha=3 \\
& \alpha=5 \\
& \alpha=7
\end{aligned}
$$

Figure 12: Effect of Womersley parameter on the shear stress $b=3, \mathrm{Ha}=1, a_{o}=3, A_{o}=2, A_{1}=4, t=$ $1, \mathrm{kn}=0.001$, and $k=0.5$.

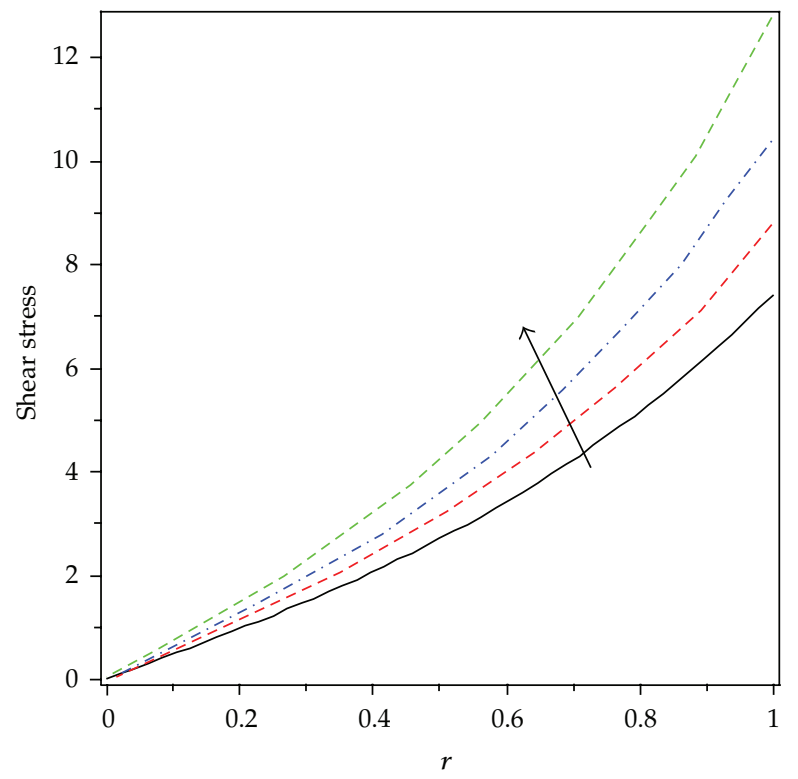

$$
\begin{aligned}
& \mathrm{kn}=0.001 \\
& \mathrm{kn}=0.1 \\
& \mathrm{kn}=0.2 \\
& \mathrm{kn}=0.3
\end{aligned}
$$

Figure 13: Effect of Knudsen numberon on the shear stress $\alpha=3$, Ha $=1, a_{o}=3, A_{o}=2, A_{1}=4, t=1, b=$ 2 , and $k=0.5$. 


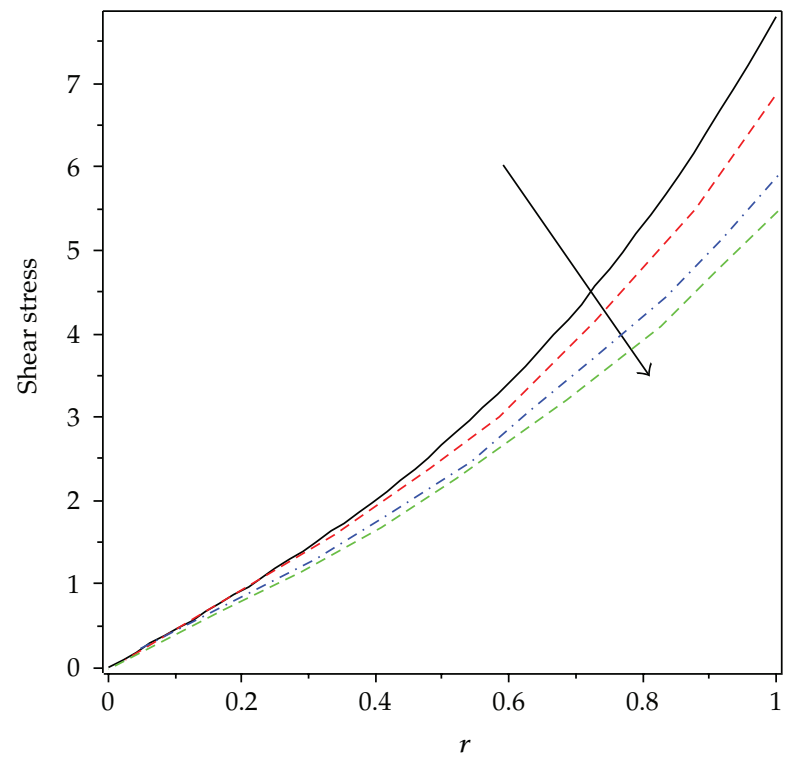

$$
\begin{aligned}
& b=1 \\
& b=2 \\
& b=3 \\
& b=4
\end{aligned}
$$

Figure 14: effect of frequency of body acceleration on the shear stress $\mathrm{Ha}=1, \alpha=3, a_{o}=3, A_{o}=2, A_{1}=4$, $t=1, \mathrm{kn}=0.2$, and $k=0.5$.

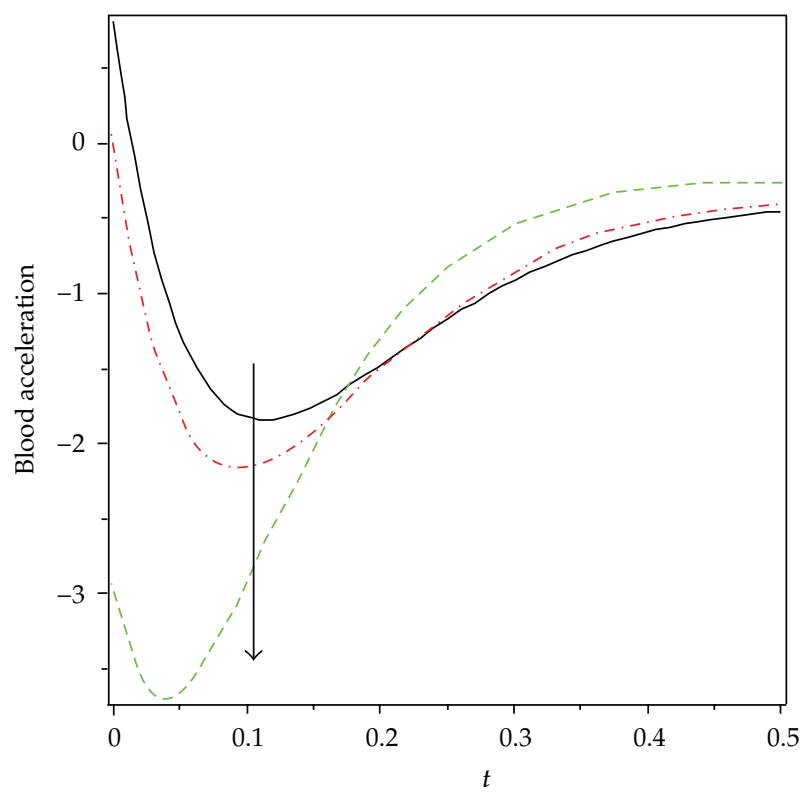

$$
\begin{aligned}
& \mathrm{Ha}=0.5 \\
& \mathrm{Ha}=1 \\
& \mathrm{Ha}=2
\end{aligned}
$$

Figure 15: Effect of Hartmann number on the blood acceleration kn $=0.001, \alpha=3, a_{o}=3, A_{o}=2, A_{1}=4$, $t=1, b=1$, and $k=0.5$. 


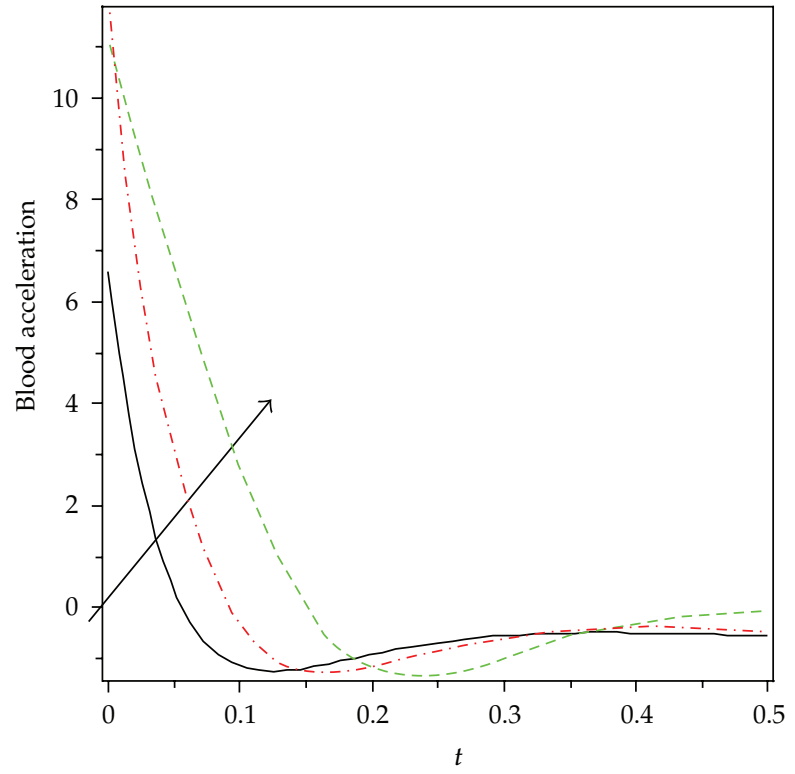

$$
\begin{aligned}
& \mathrm{kn}=0.001 \\
& \mathrm{kn}=0.1 \\
& \mathrm{kn}=0.3
\end{aligned}
$$

Figure 16: Effect of Knudsen number on the Blood acceleration $\mathrm{Ha}=1, \alpha=3, a_{o}=3, A_{o}=2, A_{1}=4$, $t=1, b=2$ and $k=0.5$.

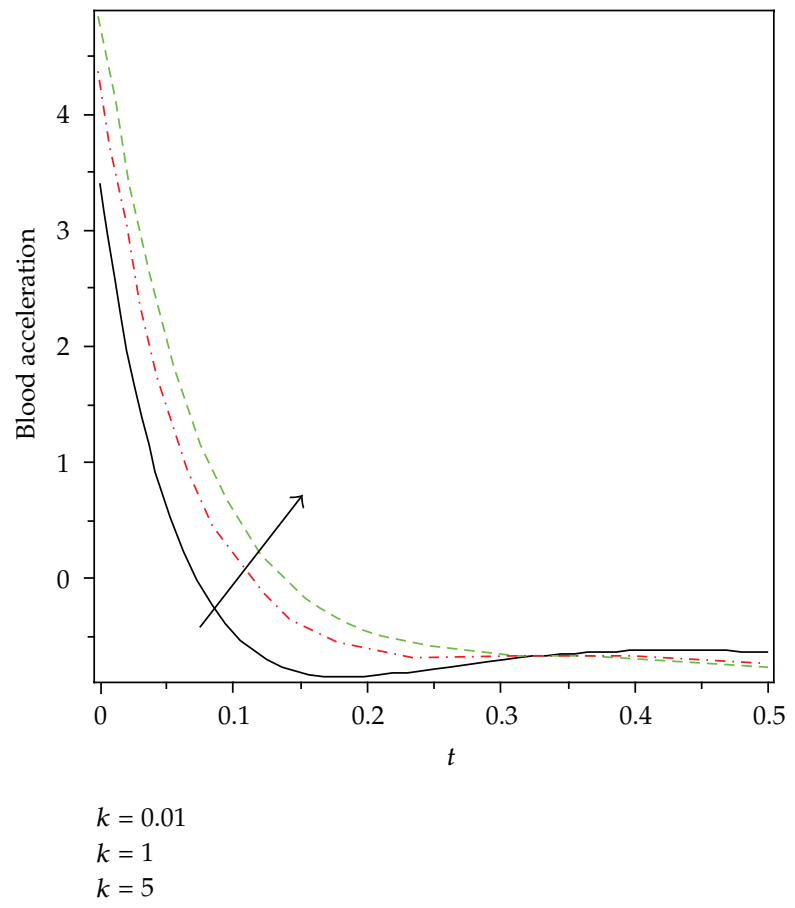

Figure 17: Effect of permeability parameter on the blood acceleration $\alpha=3$, Ha $=1, a_{o}=3, A_{o}=2$, $A_{1}=4, t=1, b=2$, and $\mathrm{kn}=0.01$. 


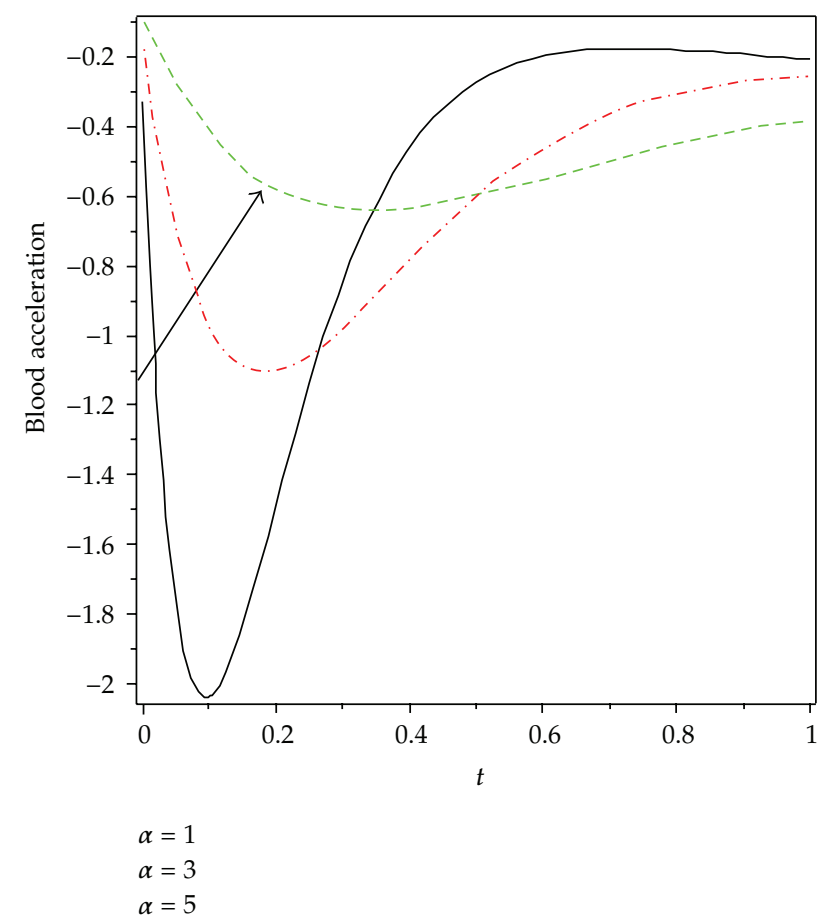

Figure 18: Effect of Womersley parameter on the blood acceleration $b=2, \mathrm{Ha}=1, a_{o}=3, A_{o}=2, A_{1}=4$, $t=1, \mathrm{kn}=0.01$, and $k=0.5$.

A numerical code has been written to calculate the axial velocity, shear stress, and fluid acceleration according to ((3.13)-(3.15)), respectively. In order to check our code, we run it for the parameters related to a realistic physical problem similar to the ones used by other authors [9, 33-36]. For instance, for $b=2, \alpha=3, a_{o}=3, A_{o}=2, A_{1}=4, t=1, k=0.5, r=0.5$, and $\mathrm{kn}=0.0$ we obtain the axial velocity $u=0.88340$, which equals (if we keep five digits after the decimal point) to the result of the authors of [34]. The same confirmation was made with the references $[1,26,33]$.

The axial velocity profile computed by using the velocity expression (3.13) for different values of Hartmann number Ha, Knudsen number kn, Womersley parameter $\alpha$, frequency of the body acceleration $b$, the permeability parameter of porous medium $k$ and have been shown through Figures 2 to 13. It is observed that from Figure 2 that as the Hartmann number increases the axial velocity decreases. Figure 3 shows that by increasing the Knudsen number the axial velocity decreases with small amount.

In Figure 4 the axial velocity of the blood increases with increasing the permeability parameter of porous medium $k$. The effect of Womersley parameter $\alpha$ on the axial elocity $u$ has been showed in Figure 5. We can see that the axial velocity increases with increasing the Womersley parameter.

Figures $6,7,8$, and 9 present the effect of the frequency of the body acceleration $b$ on the axial velocity distribution for various values of Knudsen number $\mathrm{kn}$. We note that the axial velocity decreases with increasing the frequency of body acceleration $b$. In Figure 6 we note that there is no reflux at $\mathrm{kn}=0.001$ (negative values of the axial velocity). The reflux appears in Figure 7 at $\mathrm{kn}=0.1$ the negative values begin at $r=0.9$ (near to the wall of artery) 


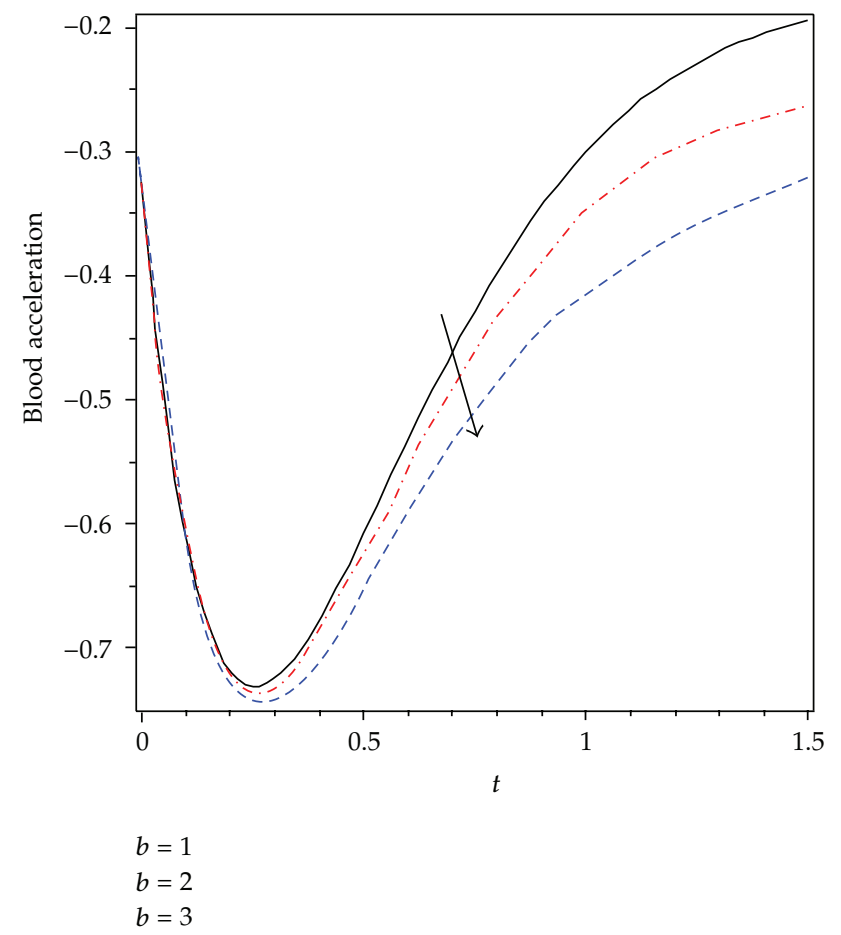

Figure 19: Effect of frequency of body acceleration on the blood acceleration $\alpha=3$, Ha $=1, a_{o}=3, A_{o}=2$, $A_{1}=4, t=1, \mathrm{kn}=0.01$, and $k=0.5$.

With increasing the value of Knudsen number $\mathrm{kn}(\mathrm{kn}=0.2)$ as in Figure 8 the reflux occurs at $r=0.6$. Whereas the reflux occurs at $r=0(\mathrm{kn}=0.3)$ as shown in Figure 9 .

The blood acceleration profile is computed by using (3.15) for different values of Hartmann number Ha, Knudsen number kn, permeability parameter of porous medium $k$, the Womersley parameter, and the frequency of the body acceleration $b$. It is observed from Figure 15 that the blood acceleration decreases with increasing the Hartmann number Ha up to $t=0.2$ and then increases with increasing the Hartmann number Ha up to $t=1$. The blood acceleration increases with increasing each of Knudsen number kn, permeability parameter of porous medium $k$ and Womersley parameter $\alpha$ up to $t=0.3$ as shown in Figures 16, 17, and 18.

The effect of Hartmann number Ha on the shear stress $\tau$ is presented in Figure 10. In all our calculations the dynamic viscosity of the blood is taken $\mu=2.5$ ref. to [9]. We note that the shear stress equals zero at the center of the artery and decreases with increasing the Hartmann number Ha. Also the shear stress $\tau$ decreases with increasing the frequency of the body acceleration $b$ as shown in Figure 14. Figures 11, 12, and 13 show that the shear stress $\tau$ increases with increasing the permeability parameter of porous medium $k$, the Womersley parameter $\alpha$ and the Knudsen number kn.

Figure 19 represents the effect of the frequency of body acceleration on the blood acceleration. We note that there is no effect (approximately) up to $t=0.4$ then the blood acceleration decreases with increasing the frequency of body acceleration. 


\section{Conclusions}

In the present mathematical model, the unsteady pulsatile blood flow through porous medium in the presence of magnetic field with periodic body acceleration through a rigid straight circular tube (artery) has been studied. The slip condition on the wall artery has been considered. The velocity expression has been obtained in an approximation way. The corresponding expressions for shear stress and fluid acceleration are also obtained. It is of interest to note that the axial velocity increases with increasing of the permeability parameter of porous medium and Womersley parameter whereas it decreases with increasing the Hartmann number, frequency of body acceleration, and Knudsen number. Also, the shear stress increases with increasing the permeability parameter of porous medium, Womersley parameter, and Knudsen number whereas decreases with increasing Hartmann number and the frequency of body acceleration. Finally, the blood acceleration increases with increasing the permeability parameter of porous medium, Womersley parameter, and Knudsen number whereas decreases with increasing Hartmann number and the frequency of body acceleration.

The present model gives a most general form of velocity expression from which the other mathematical models can easily be obtained by proper substitutions. It is of interest to note that the result of the present model includes results of different mathematical models such as:

(1) The results of Megahed et al. [34] have been recovered by taking Knudsen number $\mathrm{kn}=0.0$ (no slip condition).

(2) The results of Kamel and El-Tawil [33] have been recovered by taking Knudsen number $\mathrm{kn}=0.0$, the permeability of porous medium $k \rightarrow \infty$ without stochastic and no body acceleration.

(3) The results of El-Shahed [26] have been recoverd by taking Knudsen number $\mathrm{kn}=0.0$ and Hartmann number Ha $=0.0$ (no magnetic field).

(4) The results of Chaturani and Palanisamy [1] have been recovered by taking Knudsen number $\mathrm{kn}=0.0$, the permeability of porous medium $k \rightarrow \infty$ and Hartmann number $\mathrm{Ha}=0.0$ (no magnetic field).

It is possible that a proper understanding of interactions of body acceleration with blood flow may lead to a therapeutic use of controlled body acceleration. It is therefore desirable to analyze the effects of different types of vibrations on different parts of the body. Such a knowledge of body acceleration could be useful in the diagnosis and therapeutic treatment of some health problems (joint pain, vision loss, and vascular disorder), to better design of protective pads and machines.

By using an appropriate magnetic field it is possible to control blood pressure and also it is effective for conditions such as poor circulation, travel sickness, pain, headaches, muscle sprains, strains, and joint pains. The slip condition plays an important role in shear skin, spurt and hysteresis effects. The fluids that exhibit boundary slip have important technological applications such as in polishing valves of artificial heart and internal cavities.

Hoping that this investigation may have for further studies in the field of medical research, the application of magnetic field for the treatment of certain cardiovascular diseases, and also the results of this analysis can be applied to the pathological situations of blood flow in coronary arteries when fatty plaques of cholesterol and artery clogging blood clots are formed in the lumen of the coronary artery. 


\section{Appendix}

$$
\begin{aligned}
& M_{0}=\frac{\alpha^{2} m_{2} m_{o} \sin \left(m_{1} t\right)}{\mathrm{kn}(-1+\mathrm{kn})}, \\
& M_{1}=\frac{1}{m_{5}}+\frac{1}{16} \frac{\alpha^{2} m_{2} m_{o} \mathrm{Ha}^{2} \sin \left(m_{1} t\right)}{m_{5} \mathrm{kn}(-1+\mathrm{kn})}-\frac{\alpha^{2} m_{2} m_{o} \mathrm{Ha}^{2} \sin \left(m_{1} t\right)}{m_{5}(-1+\mathrm{kn})}-\frac{\alpha^{2} \cos \left(m_{1} t\right)}{m_{5}} \\
& +\frac{1}{2} \frac{\alpha^{2} m_{2} m_{o} \sin \left(m_{1} t\right)}{m_{5} \mathrm{kn}(-1+\mathrm{kn})}+\frac{1}{16} \frac{\alpha^{2} m_{2} m_{o} \sin \left(m_{1} t\right)}{k m_{5} k n(-1+\mathrm{kn})}, \\
& M_{2}=16 \frac{k \cos (t)}{m_{4}}+\frac{\cos (t)}{m_{4}}+64 \frac{k^{2} \cos (t)}{m_{4}}-\frac{m_{2} \cos \left(m_{1} t\right)}{m_{4}}+12 \frac{\alpha^{2} m_{2} m_{o} \mathrm{Ha}^{2} k^{2} \sin \left(m_{1} t\right)}{m_{4} \mathrm{kn}(-1+\mathrm{kn})} \\
& -6 \frac{\alpha^{2} m_{2} m_{o} \mathrm{Ha}^{2} k \sin \left(m_{1} t\right)}{m_{4}(-1+\mathrm{kn})}+\frac{3}{16} \frac{\alpha^{2} m_{2} m_{o} \mathrm{Ha}^{2} k \sin \left(m_{1} t\right)}{m_{4} \mathrm{kn}(-1+\mathrm{kn})}-\frac{m_{2} \mathrm{Ha}^{4} k^{2} \cos \left(m_{1} t\right)}{m_{4}} \\
& +2 \frac{\alpha^{2} k \sin (t)}{m_{4}}-32 \frac{\alpha^{2} \mathrm{kn} k^{2} \sin (t)}{m_{4}}+2 \frac{\alpha^{2} \mathrm{Ha}^{2} k^{2} \sin (t)}{m_{4}}+16 \frac{\alpha^{2} k^{2} \sin (t)}{m_{4}} \\
& -\frac{\alpha^{4} k^{2} \cos (t)}{m_{4}}-32 \frac{\mathrm{kn} k \cos (t)}{m_{4}}+\frac{\mathrm{Ha}^{2} k^{2} \cos (t)}{m_{4}}+16 \frac{\mathrm{Ha}^{2} k^{2} \cos (t)}{m_{4}} \\
& +2 \frac{H a^{2} k \cos (t)}{m_{4}}+32 \frac{m_{2} \mathrm{kn} k \cos \left(m_{1} t\right)}{m_{4}}-16 \frac{m_{2} k \cos \left(m_{1} t\right)}{m_{4}}+32 \frac{m_{2} \mathrm{kn} k^{2} \mathrm{Ha}^{2} \cos \left(m_{1} t\right)}{m_{4}} \\
& -32 \frac{\mathrm{kn}^{2} \mathrm{Ha}^{2} \cos \left(m_{1} t\right)}{m_{4}}+12 \frac{m_{2} k \alpha^{2} m_{o} \sin \left(m_{1} t\right)}{m_{4} \mathrm{kn}(-1+\mathrm{kn})}-3 \frac{m_{2} k^{2} \alpha^{2} m_{o} \mathrm{Ha}^{4} \sin \left(m_{1} t\right)}{m_{4} \mathrm{kn}(-1+\mathrm{kn})} \\
& +32 \frac{m_{2} k^{2} \alpha^{2} m_{o} \sin \left(m_{1} t\right)}{m_{4} k n(-1+\mathrm{kn})}-64 \frac{m_{2} k^{2} \alpha^{2} m_{o} \sin \left(m_{1} t\right)}{m_{4}(-1+\mathrm{kn})}-64 \frac{m_{2} k^{2} \cos \left(m_{1} t\right)}{m_{4}} \\
& -2 \frac{m_{2} k \mathrm{Ha}^{2} \cos \left(m_{1} t\right)}{m_{4}}+\frac{1}{16} \frac{m_{2} k \alpha^{6} m_{o} \sin \left(m_{1} t\right)}{m_{4} \mathrm{kn}(-1+\mathrm{kn})}-32 \frac{m_{2} k^{2} \alpha^{2} m_{o} \mathrm{Ha}^{2} \sin \left(m_{1} t\right)}{m_{4}(-1+\mathrm{kn})} \\
& +32 \frac{m_{2} k^{2} \alpha^{2} m_{o} \mathrm{knHa}^{2} \sin \left(m_{1} t\right)}{m_{4}(-1+\mathrm{kn})}+\frac{1}{16} \frac{m_{2} k^{2} \alpha^{6} m_{o} \mathrm{Ha}^{2} \sin \left(m_{1} t\right)}{m_{4} \mathrm{kn}(-1+\mathrm{kn})} \\
& -32 \frac{m_{2} k \alpha^{2} m_{o} \mathrm{Ha}^{2} \sin \left(m_{1} t\right)}{m_{4}(-1+\mathrm{kn})}+32 \frac{m_{2} k \alpha^{2} m_{o} \sin \left(m_{1} t\right)}{m_{4}(-1+\mathrm{kn})}-\frac{m_{2} k^{2} \alpha^{6} m_{o} \sin \left(m_{1} t\right)}{m_{4}(-1+\mathrm{kn})} \\
& +3 \frac{m_{2} k \alpha^{2} m_{\mathrm{o}} \mathrm{Ha}^{2} \sin \left(m_{1} t\right)}{m_{4} \mathrm{kn}(-1+\mathrm{kn})}+\frac{1}{16} \frac{m_{2} \alpha^{2} m_{o} \sin \left(m_{1} t\right)}{k m_{4} \mathrm{kn}(-1+\mathrm{kn})}+\frac{3}{16} \frac{m_{2} \alpha^{2} m_{\mathrm{o}} \mathrm{Ha}^{2} \sin \left(m_{1} t\right)}{m_{4} \mathrm{kn}(-1+\mathrm{kn})} \\
& -3 \frac{m_{2} \alpha^{2} m_{o} \sin \left(m_{1} t\right)}{m_{4}(-1+\mathrm{kn})}+\frac{3}{2} \frac{m_{2} \alpha^{2} m_{\mathrm{o}} \sin \left(m_{1} t\right)}{m_{4} \mathrm{kn}(-1+\mathrm{kn})}+\frac{m_{2} k^{2} \alpha^{4} \cos \left(m_{1} t\right)}{m_{4}}-16 \frac{m_{2} k^{2} \mathrm{Ha}^{2} \cos \left(m_{1} t\right)}{m_{4}} \\
& +\frac{1}{2} \frac{m_{2} \alpha^{6} k^{2} m_{o} \sin \left(m_{1} t\right)}{m_{4} \mathrm{kn}(-1+\mathrm{kn})}+\frac{1}{16} \frac{m_{2} \alpha^{2} k^{2} m_{o} \mathrm{Ha}^{6} \sin \left(m_{1} t\right)}{m_{4} \mathrm{kn}(-1+\mathrm{kn})}+\frac{3}{2} \frac{m_{2} k^{2} \alpha^{2} m_{o} \mathrm{Ha}^{4} \sin \left(m_{1} t\right)}{m_{4} \mathrm{kn}(-1+\mathrm{kn})} \\
& M_{3}=\frac{\cos (b t)}{m_{3}}+64 \frac{k^{2} \cos (b t)}{m_{3}}+16 \frac{k \cos (b t)}{m_{3}}-\frac{m_{2} \cos \left(m_{1} t\right)}{m_{3}}-3 \frac{\alpha^{2} m_{2} m_{o} \sin \left(m_{1} t\right)}{m_{3}(-1+\mathrm{kn})}
\end{aligned}
$$




$$
\begin{aligned}
& +\frac{3}{2} \frac{\alpha^{2} m_{2} m_{o} \sin \left(m_{1} t\right)}{m_{3} \mathrm{kn}(-1+\mathrm{kn})}-16 \frac{k m_{2} \cos \left(m_{1} t\right)}{m_{3}}-16 \frac{k^{2} m_{2} \mathrm{Ha}^{2} \cos \left(m_{1} t\right)}{m_{3}} \\
& +2 \frac{b k^{2} \alpha^{2} m_{2} \mathrm{Ha}^{2} \sin (b t)}{m_{3}}+\frac{1}{16} \frac{k^{2} \alpha^{2} m_{2} m_{0} H a^{6} \sin \left(m_{1} t\right)}{m_{3} k n(-1+\mathrm{kn})}+\frac{1}{16} \frac{\alpha^{2} m_{2} m_{o} \sin \left(m_{1} t\right)}{k m_{3} \mathrm{kn}(-1+\mathrm{kn})} \\
& -32 \frac{k^{2} \alpha^{2} m_{2} m_{\mathrm{o}} \mathrm{Ha}^{2} \sin \left(m_{1} t\right)}{m_{3}(-1+\mathrm{kn})}+32 \frac{k^{2} \alpha^{2} m_{2} m_{\mathrm{o}} \mathrm{knHa}^{2} \sin \left(m_{1} t\right)}{m_{3}(-1+\mathrm{kn})} \\
& -3 \frac{k^{2} \alpha^{2} m_{2} m_{o} \mathrm{Ha}^{4} \sin \left(m_{1} t\right)}{m_{3}(-1+\mathrm{kn})}-32 \frac{k \alpha^{2} m_{2} m_{o} \sin \left(m_{1} t\right)}{m_{3}(-1+\mathrm{kn})}+32 \frac{k \alpha^{2} m_{2} m_{o} \mathrm{kn} \sin \left(m_{1} t\right)}{m_{3}(-1+\mathrm{kn})} \\
& -\frac{k^{2} \alpha^{6} m_{2} m_{o} b^{2} \sin \left(m_{1} t\right)}{m_{3}(-1+\mathrm{kn})}+3 \frac{k \alpha^{2} m_{2} m_{o} \mathrm{Ha}^{2} \sin \left(m_{1} t\right)}{m_{3} \mathrm{kn}(-1+\mathrm{kn})}+12 \frac{k \alpha^{2} m_{2} m_{o} \sin \left(m_{1} t\right)}{m_{3} \mathrm{kn}(-1+k n)} \\
& -32 \frac{b k^{2} \alpha^{2} \mathrm{kn} \sin (b t)}{m_{3}}+\frac{3}{16} \frac{\alpha^{2} m_{2} m_{o} \mathrm{Ha}^{2} \sin \left(m_{1} t\right)}{m_{3} k n(-1+\mathrm{kn})}+\frac{3}{2} \frac{k^{2} \alpha^{2} m_{2} m_{o} \mathrm{Ha}^{4} \sin \left(m_{1} t\right)}{m_{3} k n(-1+\mathrm{kn})} \\
& +12 \frac{k^{2} \alpha^{2} m_{2} m_{o} \mathrm{Ha}^{2} \sin \left(m_{1} t\right)}{m_{3} \mathrm{kn}(-1+\mathrm{kn})}-6 \frac{k \alpha^{2} m_{2} m_{\mathrm{o}} \mathrm{Ha}^{2} \sin \left(m_{1} t\right)}{m_{3}(-1+\mathrm{kn})} \\
& +\frac{3}{16} \frac{k \alpha^{2} m_{2} m_{o} \mathrm{Ha}^{4} \sin \left(m_{1} t\right)}{m_{3} \mathrm{kn}(-1+\mathrm{kn})}+32 \frac{k^{2} \alpha^{2} m_{2} m_{o} \sin \left(m_{1} t\right)}{m_{3} \mathrm{kn}(-1+\mathrm{kn})}+32 \frac{k m_{2} \mathrm{kn} \cos \left(m_{1} t\right)}{m_{3}} \\
& +32 \frac{k^{2} m_{2} \mathrm{knHa}^{2} \cos \left(m_{1} t\right)}{m_{3}}-64 \frac{k^{2} \alpha^{2} m_{2} m_{o} \sin \left(m_{1} t\right)}{m_{3}(-1+\mathrm{kn})}-\frac{k^{2} m_{2} \mathrm{Ha}^{4} \cos \left(m_{1} t\right)}{m_{3}} \\
& +2 \frac{b k \alpha^{2} \sin (b t)}{m_{3}}+16 \frac{k^{2} \mathrm{Ha}^{2} \cos (b t)}{m_{3}}-32 \frac{k \mathrm{kn} \cos (b t)}{m_{3}}+2 \frac{k \mathrm{Ha}^{2} \cos (b t)}{m_{3}} \\
& +\frac{k^{2} \mathrm{Ha}^{4} \cos (b t)}{m_{3}}-32 \frac{k^{2} \mathrm{knHa}^{2} \cos (b t)}{m_{3}}-\frac{k^{2} \alpha^{4} b^{2} \cos (b t)}{m_{3}}+16 \frac{b k^{2} \alpha^{2} \sin (b t)}{m_{3}} \\
& +\frac{k^{2} \alpha^{4} b^{2} m_{2} \cos \left(m_{1} t\right)}{m_{3}}+\frac{1}{16} \frac{k^{2} \alpha^{6} m_{2} m_{o} \mathrm{Ha}^{2} b^{2} \sin \left(m_{1} t\right)}{m_{3} \mathrm{kn}(-1+k n)} \\
& +\frac{1}{2} \frac{k^{2} \alpha^{6} m_{2} m_{o} b^{2} \sin \left(m_{1} t\right)}{m_{3} \mathrm{kn}(-1+\mathrm{kn})}-2 \frac{k m_{2} \mathrm{Ha}^{2} \cos \left(m_{1} t\right)}{m_{3}}+\frac{1}{16} \frac{k \alpha^{6} m_{2} m_{o} b^{2} \sin \left(m_{1} t\right)}{m_{3} \mathrm{kn}(-1+\mathrm{kn})}, \\
& M_{4}=\frac{1}{2} \frac{m_{2} m_{o} \sin \left(m_{1} t\right)}{k \mathrm{kn}(-1+\mathrm{kn})}-\frac{m_{2} \cos \left(m_{1} t\right)}{k \alpha^{2}(-1+\mathrm{kn})}+\frac{m_{2} \mathrm{kn} \cos \left(m_{1} t\right)}{k \alpha^{2}(-1+\mathrm{kn})}-\frac{m_{2} m_{o} \sin \left(m_{1} t\right)}{k(-1+\mathrm{kn})}, \\
& M_{5}=\frac{\mathrm{Ha}^{2} k}{m_{5}}+\frac{1}{m_{5}}+\frac{m_{2} \cos \left(m_{1} t\right)}{m_{5}(-1+\mathrm{kn})}-\frac{m_{2} \mathrm{kn} \cos \left(m_{1} t\right)}{m_{5}(-1+\mathrm{kn})}-\frac{k m_{2} \mathrm{knHa}^{2} \cos \left(m_{1} t\right)}{m_{5}(-1+\mathrm{kn})} \\
& +\frac{\alpha^{2} m_{2} m_{o} \sin \left(m_{1} t\right)}{m_{5}(-1+\mathrm{kn})}+\frac{k m_{2} \mathrm{Ha}^{2} \cos \left(m_{1} t\right)}{m_{5}(-1+\mathrm{kn})}+8 \frac{k m_{2} \cos \left(m_{1} t\right)}{m_{5}}-16 \frac{k m_{2} \mathrm{kn} \cos \left(m_{1} t\right)}{m_{5}} \\
& +8 \frac{k m_{2} \cos \left(m_{1} t\right)}{m_{5}(-1+\mathrm{kn})}-\frac{1}{2} \frac{k \alpha^{2} m_{2} m_{\mathrm{o}} \mathrm{Ha}^{2} \sin \left(m_{1} t\right)}{m_{5} \mathrm{kn}(-1+\mathrm{kn})}+\frac{k \alpha^{2} m_{2} m_{o} \mathrm{Ha}^{2} \sin \left(m_{1} t\right)}{m_{5}(-1+\mathrm{kn})}
\end{aligned}
$$




$$
\begin{aligned}
& -4 \frac{k \alpha^{2} m_{2} m_{o} \sin \left(m_{1} t\right)}{m_{5} \mathrm{kn}(-1+\mathrm{kn})}-24 \frac{k m_{2} \mathrm{kn} \cos \left(m_{1} t\right)}{m_{5}(-1+\mathrm{kn})}+16 \frac{k m_{2} \mathrm{kn} \cos \left(m_{1} t\right)}{m_{5}(-1+\mathrm{kn})} \\
& -\frac{1}{2} \frac{\alpha^{2} m_{2} m_{o} \sin \left(m_{1} t\right)}{m_{5} \mathrm{kn}(-1+\mathrm{kn})} \\
& M_{6}=16 \frac{k \cos (t)}{m_{4}}+\frac{\alpha^{4} \mathrm{Ha}^{2} k^{3} \cos (t)}{m_{4}}+64 \frac{k^{2} \cos (t)}{m_{4}}-\frac{m_{2} \cos \left(m_{1} t\right)}{m_{4}} \\
& +12 \frac{\alpha^{2} m_{2} m_{o} \mathrm{Ha}^{2} k^{2} \sin \left(m_{1} t\right)}{m_{4} k n(-1+\mathrm{kn})} \\
& -6 \frac{\alpha^{2} m_{2} m_{o} \mathrm{Ha}^{2} k \sin \left(m_{1} t\right)}{m_{4}(-1+\mathrm{kn})}+\frac{3}{16} \frac{\alpha^{2} m_{2} m_{o} \mathrm{Ha}^{2} k \sin \left(m_{1} t\right)}{m_{4} \mathrm{kn}(-1+\mathrm{kn})}-\frac{m_{2} \mathrm{Ha}^{4} k^{2} \cos \left(m_{1} t\right)}{m_{4}} \\
& +2 \frac{\alpha^{2} k \sin (t)}{m_{4}}-32 \frac{\alpha^{2} \mathrm{kn} k^{2} \sin (t)}{m_{4}}+2 \frac{\alpha^{2} \mathrm{Ha}^{2} k^{2} \sin (t)}{m_{4}}+16 \frac{\alpha^{2} k^{2} \sin (t)}{m_{4}} \\
& -\frac{\alpha^{4} k^{2} \cos (t)}{m_{4}}-32 \frac{\mathrm{kn} k \cos (t)}{m_{4}}+\frac{\mathrm{Ha}^{4} k^{2} \cos (t)}{m_{4}}+16 \frac{\mathrm{Ha}^{2} k^{2} \cos (t)}{m_{4}} \\
& +2 \frac{\mathrm{Ha}^{2} k \cos (t)}{m_{4}}+32 \frac{m_{2} \mathrm{kn} k \cos \left(m_{1} t\right)}{m_{4}}-16 \frac{m_{2} k \cos \left(m_{1} t\right)}{m_{4}}+32 \frac{m_{2} \mathrm{kn}^{2} \mathrm{Ha}^{2} \cos \left(m_{1} t\right)}{m_{4}} \\
& -32 \frac{\mathrm{kn}^{2} \mathrm{Ha}^{2} \cos \left(m_{1} t\right)}{m_{4}}+12 \frac{m_{2} k \alpha^{2} m_{o} \sin \left(m_{1} t\right)}{m_{4} \mathrm{kn}(-1+\mathrm{kn})}-3 \frac{m_{2} k^{2} \alpha^{2} m_{o} \mathrm{Ha}^{4} \sin \left(m_{1} t\right)}{m_{4} \mathrm{kn}(-1+\mathrm{kn})} \\
& +32 \frac{m_{2} k^{2} \alpha^{2} m_{o} \sin \left(m_{1} t\right)}{m_{4} k n(-1+k n)}-64 \frac{m_{2} k^{2} \alpha^{2} m_{o} \sin \left(m_{1} t\right)}{m_{4}(-1+k n)}-64 \frac{m_{2} k^{2} \cos \left(m_{1} t\right)}{m_{4}} \\
& -2 \frac{m_{2} k \mathrm{Ha}^{2} \cos \left(m_{1} t\right)}{m_{4}}+\frac{1}{16} \frac{m_{2} k \alpha^{6} m_{o} \sin \left(m_{1} t\right)}{m_{4} \mathrm{kn}(-1+\mathrm{kn})}-32 \frac{m_{2} k^{2} \alpha^{2} m_{o} \mathrm{Ha}^{2} \sin \left(m_{1} t\right)}{m_{4}(-1+\mathrm{kn})} \\
& +32 \frac{m_{2} k^{2} \alpha^{2} m_{o} \mathrm{knHa}^{2} \sin \left(m_{1} t\right)}{m_{4}(-1+\mathrm{kn})}+\frac{1}{16} \frac{m_{2} k^{2} \alpha^{6} m_{o} \mathrm{Ha}^{2} \sin \left(m_{1} t\right)}{m_{4} \mathrm{kn}(-1+\mathrm{kn})} \\
& -32 \frac{m_{2} k \alpha^{2} m_{o} \mathrm{Ha}^{2} \sin \left(m_{1} t\right)}{m_{4}(-1+\mathrm{kn})}+32 \frac{m_{2} k \alpha^{2} m_{o} \sin \left(m_{1} t\right)}{m_{4}(-1+\mathrm{kn})}-\frac{m_{2} k^{2} \alpha^{6} m_{o} \sin \left(m_{1} t\right)}{m_{4}(-1+\mathrm{kn})} \\
& +3 \frac{m_{2} k \alpha^{2} m_{o} \mathrm{Ha}^{2} \sin \left(m_{1} t\right)}{m_{4} \mathrm{kn}(-1+\mathrm{kn})}+\frac{1}{16} \frac{m_{2} \alpha^{2} m_{o} \sin \left(m_{1} t\right)}{k m_{4} \mathrm{kn}(-1+\mathrm{kn})}+\frac{3}{16} \frac{m_{2} \alpha^{2} m_{o} \mathrm{Ha}^{2} \sin \left(m_{1} t\right)}{m_{4} \mathrm{kn}(-1+\mathrm{kn})} \\
& -3 \frac{m_{2} \alpha^{2} m_{o} \sin \left(m_{1} t\right)}{m_{4}(-1+\mathrm{kn})}+\frac{3}{2} \frac{m_{2} \alpha^{2} m_{o} \sin \left(m_{1} t\right)}{m_{4} \mathrm{kn}(-1+\mathrm{kn})}+\frac{m_{2} k^{2} \alpha^{4} \cos \left(m_{1} t\right)}{m_{4}} \\
& -16 \frac{m_{2} k^{2} \mathrm{Ha}^{2} \cos \left(m_{1} t\right)}{m_{4}} \\
& M_{7}=\frac{k \cos (b t)}{m_{4}}+16 \frac{\alpha^{4} \mathrm{Ha}^{2} k^{3} b^{2} \cos \left(m_{1} t\right)}{m_{4}}+8 \frac{m_{2} \cos \left(m_{1} t\right)}{m_{4}}
\end{aligned}
$$




$$
\begin{aligned}
& +12 \frac{\alpha^{6} m_{2} m_{0} \mathrm{Ha}^{2} k^{2} b^{2} \sin \left(m_{1} t\right)}{m_{4} \mathrm{kn}(-1+\mathrm{kn})} \\
& -6 \frac{\alpha^{2} m_{2} m_{\mathrm{o}} \mathrm{Ha}^{2} k \sin \left(m_{1} t\right)}{m_{4}(-1+\mathrm{kn})}+\frac{3}{16} \frac{\alpha^{2} m_{2} m_{\mathrm{o}} \mathrm{Ha}^{2} k \sin \left(m_{1} t\right)}{m_{4} \mathrm{kn}(-1+\mathrm{kn})}-\frac{m_{2} \mathrm{Ha}^{4} k^{2} \cos \left(m_{1} t\right)}{m_{4}} \\
& +2 \frac{\alpha^{2} k \sin (t)}{m_{4}}-32 \frac{\alpha^{2} \mathrm{kn} k^{2} \sin (t)}{m_{4}}+2 \frac{\alpha^{2} \mathrm{Ha}^{2} k^{2} \sin (t)}{m_{4}}+16 \frac{\alpha^{2} k^{2} \sin (t)}{m_{4}} \\
& -\frac{\alpha^{4} k^{2} \cos (t)}{m_{4}}-32 \frac{\mathrm{kn} k \cos (t)}{m_{4}}+\frac{\mathrm{Ha}^{4} k^{2} \cos (t)}{m_{4}}+16 \frac{\mathrm{Ha}^{2} k^{2} \cos (t)}{m_{4}} \\
& +2 \frac{\mathrm{Ha}^{2} k \cos (t)}{m_{4}}+32 \frac{m_{2} \mathrm{kn} k \cos \left(m_{1} t\right)}{m_{4}}-16 \frac{m_{2} k \cos \left(m_{1} t\right)}{m_{4}}+32 \frac{m_{2} \mathrm{knk}^{2} \mathrm{Ha}^{2} \cos \left(m_{1} t\right)}{m_{4}} \\
& -\frac{\mathrm{kn}^{2} \mathrm{Ha}^{2} \cos \left(m_{1} t\right)}{m_{4}}+12 \frac{m_{2} k \alpha^{2} m_{o} \sin \left(m_{1} t\right)}{m_{4} \mathrm{kn}(-1+\mathrm{kn})}-3 \frac{m_{2} k^{2} \alpha^{2} m_{o} \mathrm{Ha}^{4} \sin \left(m_{1} t\right)}{m_{4} \mathrm{kn}(-1+\mathrm{kn})} \\
& +\frac{m_{2} k^{2} \alpha^{2} m_{o} \sin \left(m_{1} t\right)}{m_{4} \mathrm{kn}(-1+\mathrm{kn})}-64 \frac{m_{2} k^{2} \alpha^{2} m_{o} \sin \left(m_{1} t\right)}{m_{4}(-1+k n)}-64 \frac{m_{2} k^{2} \cos \left(m_{1} t\right)}{m_{4}} \\
& -2 \frac{m_{2} k \mathrm{Ha}^{2} \cos \left(m_{1} t\right)}{m_{4}}+\frac{1}{16} \frac{m_{2} k \alpha^{6} m_{o} \sin \left(m_{1} t\right)}{m_{4} k n(-1+\mathrm{kn})}-32 \frac{m_{2} k^{2} \alpha^{2} m_{\mathrm{o}} \mathrm{Ha}^{2} \sin \left(m_{1} t\right)}{m_{4}(-1+\mathrm{kn})} \\
& +\frac{m_{2} k^{2} \alpha^{2} m_{o} \mathrm{knHa}^{2} \sin \left(m_{1} t\right)}{m_{4}(-1+\mathrm{kn})}+\frac{1}{16} \frac{m_{2} k^{2} \alpha^{6} m_{o} \mathrm{Ha}^{2} \sin \left(m_{1} t\right)}{m_{4} \mathrm{kn}(-1+\mathrm{kn})} \\
& -\frac{m_{2} k \alpha^{2} m_{o} \mathrm{kn} \sin \left(m_{1} t\right)}{m_{4}(-1+\mathrm{kn})}+32 \frac{m_{2} k \alpha^{2} m_{O} \sin \left(m_{1} t\right)}{m_{4}(-1+\mathrm{kn})}-\frac{m_{2} k^{2} \alpha^{6} m_{o} \sin \left(m_{1} t\right)}{m_{4}(-1+\mathrm{kn})} \\
& +\frac{m_{2} k \alpha^{2} m_{o} H a^{2} \sin \left(m_{1} t\right)}{m_{4} \mathrm{kn}(-1+\mathrm{kn})}+\frac{1}{16} \frac{m_{2} \alpha^{2} m_{o} \sin \left(m_{1} t\right)}{k m_{4} \mathrm{kn}(-1+\mathrm{kn})}+\frac{3}{16} \frac{m_{2} \alpha^{2} m_{o} \mathrm{Ha}^{2} \sin \left(m_{1} t\right)}{m_{4} \mathrm{kn}(-1+\mathrm{kn})} \\
& -\frac{m_{2} \alpha^{2} m_{o} \sin \left(m_{1} t\right)}{m_{4}(-1+\mathrm{kn})}+\frac{3}{2} \frac{m_{2} \alpha^{2} m_{o} \sin \left(m_{1} t\right)}{m_{4} \mathrm{kn}(-1+\mathrm{kn})}+\frac{m_{2} k^{2} \alpha^{4} \cos \left(m_{1} t\right)}{m_{4}}-16 \frac{m_{2} k^{2} \mathrm{Ha}^{2} \cos \left(m_{1} t\right)}{m_{4}} \\
& +\frac{b^{2} \alpha^{4} k^{2} m_{o} \cos (b t)}{m_{3}}+128 \frac{m_{2} k^{2} \cos \left(m_{1} t\right)}{m_{3}}-\frac{1}{2} \frac{m_{2} k^{2} \alpha^{2} m_{o} b^{2} \sin \left(m_{1} t\right)}{m_{3} \mathrm{kn}(-1+\mathrm{kn})}, \\
& m_{o}=\sqrt{-\frac{\mathrm{kn}(-1+\mathrm{kn})}{\alpha^{4}}} \\
& m_{1}=16 m_{0} \\
& m_{2}=e^{\left(-(1 / 2)\left(\left(16 k^{2} \alpha^{2}-32 \mathrm{kn} k^{2} \alpha^{2}+2 k \alpha^{2}+2 k^{2} \alpha^{2} \mathrm{Ha}^{2}\right) t / k^{2} \alpha^{4}\right)\right)}, \\
& m_{3}=1+32 k+384 k^{4} \mathrm{Ha}^{4}+32 k^{4} \mathrm{Ha}^{6}+k^{4} \mathrm{Ha}^{8}+2048 k^{4} \mathrm{Ha}^{2}+4 k^{3} \mathrm{Ha}^{6}+96 k^{3} \mathrm{Ha}^{4} \\
& -4096 k^{3} \mathrm{kn}+768 k^{3} \mathrm{Ha}^{2}-192 \mathrm{knHa}{ }^{2} k^{2}+4 \mathrm{Ha}^{2} k+96 \mathrm{Ha}^{2} k^{2}-64 k \mathrm{kn}+6 \mathrm{Ha}^{4} k^{2} \\
& -4096 \mathrm{Ha}^{2} k^{4} \mathrm{kn}+2048 k^{3}-64 \mathrm{Ha}^{6} k^{4} \mathrm{kn}+4096 k^{4}+1024 \mathrm{Ha}^{4} k^{4} \mathrm{kn}^{2}+384 k^{2}
\end{aligned}
$$




$$
\begin{aligned}
+ & 4 \mathrm{Ha}^{2} k^{3} b^{3} \alpha^{4}+2 \mathrm{Ha}^{4} k^{4} b^{2} \alpha^{4}-2048 \mathrm{knHa}{ }^{2} k^{3}+2048 \mathrm{kn}^{2} \mathrm{Ha}^{2} k^{3}+32 k^{3} b^{2} \alpha^{4} \\
+ & 128 k^{4} b^{2} \alpha^{4}-64 k^{3} b^{2} \alpha^{4} \mathrm{kn}-1024 \mathrm{knHa}{ }^{4} k^{4}-64 \mathrm{knHa}{ }^{2} k^{4} b^{2} \alpha^{4}+1024 k^{4} b^{2} \alpha^{4} \mathrm{kn}^{2} \\
+ & 32 \mathrm{Ha}^{2} k^{4} b^{2} \alpha^{4}+k^{4} b^{4} \alpha^{8}-1024 k^{4} b^{2} \alpha^{4} \mathrm{kn}-192 \mathrm{knHa}{ }^{4} k^{3}+2 k^{2} b^{2} \alpha^{4} \\
- & 1024 \mathrm{kn} k^{2}+1024 k^{2} \mathrm{kn}^{2}, \\
m_{4}= & +32 k+384 k^{4} \mathrm{Ha}^{4}+32 k^{4} \mathrm{Ha}^{6}+k^{4} \mathrm{Ha}^{8}+128 k^{4} \alpha^{6} \\
& +2048 k^{4} \mathrm{Ha}^{2}+32 k^{3} \alpha^{4}+4 k^{3} \mathrm{Ha}^{6} \\
+ & 96 k^{3} \mathrm{Ha}^{4}-4096 k^{3} \mathrm{kn}+768 k^{3} \mathrm{Ha}^{2}-192 \mathrm{kn} k^{2} \mathrm{Ha}^{2} \\
+ & 4 k \mathrm{Ha}^{2}+96 k^{2} \mathrm{Ha}^{2}-64 k \mathrm{kn}+6 k^{2} \mathrm{Ha}^{4} \\
+ & 1024 k^{4} \mathrm{Ha}^{4} \mathrm{kn}^{2}-64 k^{3} \alpha^{4} \mathrm{kn}-4096 k^{4} \mathrm{Ha}^{2} \mathrm{kn}+2048 k^{3}-64 k^{4} \mathrm{Ha}^{6} \mathrm{kn}+4096 k^{4} \\
+ & 1024 k^{4} \mathrm{Ha}^{4} \mathrm{kn}^{2}+32 k^{4} \alpha^{4} \mathrm{Ha}^{2}-64 k^{4} \mathrm{Ha}^{2} \alpha^{2} \mathrm{kn}+384 k^{2}+2 k^{2} \alpha^{4}-2048 k^{3} \mathrm{Ha}^{2} \mathrm{kn} \\
+ & 2048 k^{3} \mathrm{Ha}^{2} \mathrm{kn}^{2}+4 k^{3} \mathrm{Ha}^{2} \alpha^{4}-1024 k^{4} \mathrm{Ha}^{4} \mathrm{kn} \\
& -192 k^{3} \mathrm{Ha}^{4} \mathrm{kn}^{2}+2 k^{4} \mathrm{Ha}^{4} \alpha^{4}+k^{4} \alpha^{8} \\
& -1024 k^{4} \alpha^{4} \mathrm{kn}^{2} 1024 \mathrm{kn}^{2}+1024 k^{2} \mathrm{kn}^{2}, \\
& m_{5}=64 k^{2}-32 k^{2} \mathrm{Ha}^{2} \mathrm{kn}^{2}+16 k^{2} \mathrm{Ha}^{2}+16 k+1-32 k \mathrm{kn}+2 k \mathrm{Ha}^{2}+k^{2} \mathrm{Ha}^{4} .
\end{aligned}
$$

\section{Acknowledgment}

The author would like to express deep thanks to referee for providing valuable suggestions to improve the quality of the paper.

\section{References}

[1] P. Chaturani and V. Palanisamy, "Pulsatile flow of blood with periodic body acceleration," International Journal of Engineering Science, vol. 29, no. 1, pp. 113-121, 1991.

[2] S. A. N. Elsoud, S. F. Kaldas, and S. K. Mekheimer, "Interaction of peristaltic flow with pulsatile couple stress fluid," Journal of Biomathematics, vol. 13, no. 4, pp. 417-425, 1998.

[3] E. F. Elshehawey, E. M. E. Elbarbary, N. A. S. Afifi, and M. El-Shahed, "Pulsatile flow of blood through a porous medium under periodic body acceleration," International Journal of Theoretical Physics, vol. 39, no. 1, pp. 183-188, 2000.

[4] K. Das and G. C. Saha, "Arterial MHD pulsatile flow of blood under periodic body acceleration," Bulletin of Society of Mathematicians. Banja Luka, vol. 16, pp. 21-42, 2009.

[5] D. C. Sanyal and A. Biswas, "Pulsatile motion of blood through an axi-symmetric artery in presence of magnetic field," Assam University Journal of Science and Technology: Physical Sciences and Technology, vol. 5, no. 2, pp. 12-20, 2010.

[6] G. P. Rao, S. Ravikumar, C. Vasudev, and R. S. Prasad, "Unsteady two-phase viscous ideal fluid flow through a parallel plate channel under a pulsatile pressure gradient subjected to a body acceleration," Journal of Engineering and Applied Sciences, vol. 6, no. 1, pp. 1-7, 2011. 
[7] K. S. Mekheimer, "Peristaltic flow of blood under effect of a magnetic field in a non-uniform channels," Applied Mathematics and Computation, vol. 153, no. 3, pp. 763-777, 2004.

[8] E. E. Tzirtzilakis, "A mathematical model for blood flow in magnetic field," Physics of Fluids, vol. 17, no. 7, Article ID 077103, 15 pages, 2005.

[9] M. Jain, G. C. Sharma, and A. Singh, "Mathematical analysis of mhd flow of blood in very narrow capillaries," International Journal of Engineering, Transactions B, vol. 22, no. 3, pp. 307-315, 2009.

[10] N. Verma and R. S. Parihar, "Effects of Magneto-hydrodynamic and hematocrit on blood flow in an artery with multiple mild stenosis," International Journal of Applied Mathematics and Computation, vol. 1, no. 1, pp. 30-46, 2009.

[11] J. Singh and R. Rathee, "Analytical solution of two-dimensional model of blood flow with variable viscosity through an indented artery due to LDL effect in the presence of magnetic field," International Journal of Physical Sciences, vol. 5, no. 12, pp. 1857-1868, 2010.

[12] A. R. A. Khaled and K. Vafai, "The role of porous media in modeling flow and heat transfer in biological tissues," International Journal of Heat and Mass Transfer, vol. 46, no. 26, pp. 4989-5003, 2003.

[13] A. Sinha, J. C. Misra, and G. C. Shit, "Mathematical modeling of blood flow in a porous vessel having double stenoses in the presence of an external magnetic field," International Journal of Biomathematics, vol. 4, no. 2, pp. 207-225, 2011.

[14] G. Ramamurthy and B. Shanker, "Magnetohydrodynamic effects on blood flow through a porous channel," Medical and Biological Engineering and Computing, vol. 32, no. 6, pp. 655-659, 1994.

[15] I. M. Eldesoky and A. A. Mousa, "Peristaltic flow of a compressible non-Newtonian Maxwellian fluid through porous medium in a tube," International Journal of Biomathematics, vol. 3, no. 2, pp. 255-275, 2010.

[16] G. R. Reddy and S. Venkataramana, "Peristaltic transport of a conducting fluid through a porous medium in an asymmetric vertical channel," Journal of Advances in Applied Sciences, vol. 2, no. 5, pp. 240-248, 2011.

[17] T. Hayat, Q. Hussain, and N. Ali, "Influence of partial slip on the peristaltic flow in a porous medium," Physica A, vol. 387, no. 14, pp. 3399-3409, 2008.

[18] E. F. El-Shehawy, N. T. El-Dabe, and I. M. El-Desoky, "Slip effects on the peristaltic flow of a nonNewtonian Maxwellian fluid," Acta Mechanica, vol. 186, no. 1-4, pp. 141-159, 2006.

[19] I. M. Eldesoky, "influence of slip condition on peristaltic transport of a compressible Maxwell fluid through porous medium in a tube," International Journal of Applied Mathematics and Mechanics, vol. 8, no. 2, pp. 99-117, 2012.

[20] W. Kwang-Hua Chu and J. Fang, "Peristaltic transport in a slip flow," European Physical Journal B, vol. 16, no. 3, pp. 543-547, 2000.

[21] N. Ali, Q. Hussain, T. Hayat, and S. Asghar, "Slip effects on the peristaltic transport of MHD fluid with variable viscosity," Physics Letters A, vol. 372, no. 9, pp. 1477-1489, 2008.

[22] T. Hayat, M. Umar, and N. Ali, "The influence of slip on the peristaltic motion of a third order fluid in an asymmetric channel," Physics Letters A, vol. 372, no. 15, pp. 2653-2664, 2008.

[23] A. M. Sobh, "Interaction of couple stresses and slip flow on peristaltic transport in uniform and nonuniform channels," Turkish Journal of Engineering and Environmental Sciences, vol. 32, no. 2, pp. 117-123, 2008.

[24] A. M. Sobh, "Slip flow in peristaltic transport of a Carreau fluid in an asymmetric channel," Canadian Journal of Physics, vol. 87, no. 8, pp. 957-965, 2009.

[25] S. Nadeem and S. Akram, "Slip effects on the peristaltic flow of a Jeffrey fluid in an asymmetric channel under the effect of induced magnetic field," International Journal for Numerical Methods in Fluids, vol. 63, no. 3, pp. 374-394, 2010.

[26] M. El-Shahed, "Pulsatile flow of blood through a stenosed porous medium under periodic body acceleration," Applied Mathematics and Computation, vol. 138, no. 2-3, pp. 479-488, 2003.

[27] E. F. Elshehawey, E. M. E. Elbarbary, A. S. N. Elsoud, and M. Elshahed, "Blood flow through a porous medium under periodic body acceleration," Mathematical Sciences Research Hot-Line, vol. 11, pp. 27-31, 1998.

[28] E. F. Elshehawey, E. M. Elbarabry, A. S. N. Elsoud, and M. Elshahed, “Womersley problem for pulsatile flow of blood through a porous medium," in Proceedings of the 6th Conference on Theoretical and Applied Mechanics, pp. 115-122, Cairo, Egypt, March 1999.

[29] E. F. Elshehawey, E. M. E. Elbarbary, N. A. S. Afifi, and M. El-Shahed, "MHD flow of blood under body acceleration," Integral Transforms and Special Functions, vol. 12, no. 1, pp. 1-6, 2001. 
[30] E. F. El-Shehawey, E. M. E. Elbarbary, N. A. S. Afifi, and M. Elshahed, "MHD flow of an elasticoviscous fluid under periodic body acceleration," International Journal of Mathematics and Mathematical Sciences, vol. 23, no. 11, pp. 795-799, 2000.

[31] C. Y. Wang, "Stagnation flows with slip: exact solutions of the Navier-Stokes equations," Zeitschrift fur Angewandte Mathematik und Physik, vol. 54, no. 1, pp. 184-189, 2003.

[32] C. J. Tranter, Bessel Functions with Some Physical Applications, Hart Publishing, New York, NY, USA, 1968, Printed in Great Britain.

[33] M. H. Kamel and M. A. El-Tawil, "Stochastic blood flow through an overlapping arterial stenosis," Journal of Engineering and Applied Science, vol. 48, no. 4, pp. 623-635, 2001.

[34] A. A. Megahed, B. M. Maher, and I. M. Eldesoky, “Unsteady MHD pulsatile flow through porous medium in an infinite circular pipe under the effect of the body acceleration," Journal of Science Bulletin Faculty of Engineering, Ain Shams University, vol. 39, no. 4, pp. 715-735, 2004.

[35] V. P. Rathod and T. Shakera, "Pulsatile flow of couple stress fluid through a porous medium with periodic body acceleration and magnetic field," Bulletin of the Malaysian Mathematical Sciences Society, vol. 32, no. 2, pp. 245-259, 2009.

[36] E. David and E. Shmuel, Standered Handbook of Biomedical Engineering and Design, McGraw-Hill, New York, NY, USA, 2004. 


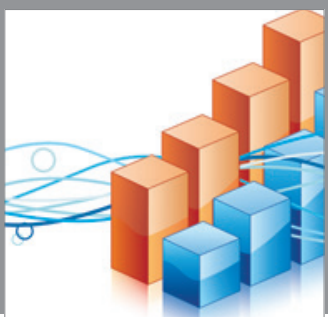

Advances in

Operations Research

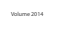

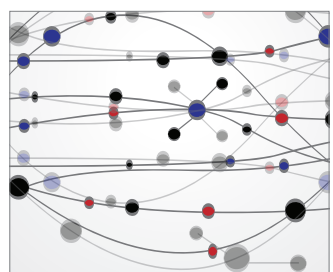

\section{The Scientific} World Journal
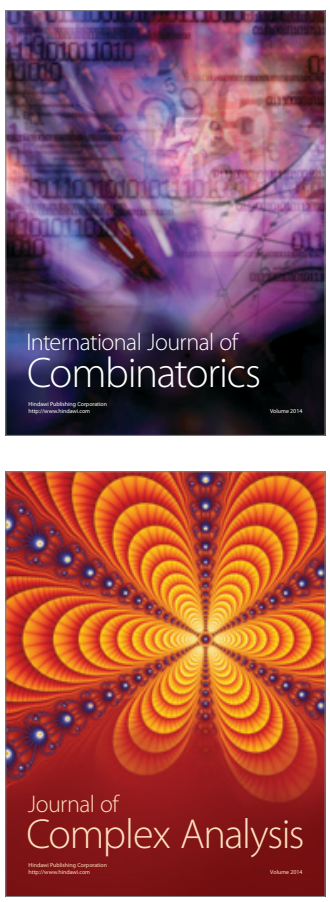

International Journal of

Mathematics and

Mathematical

Sciences
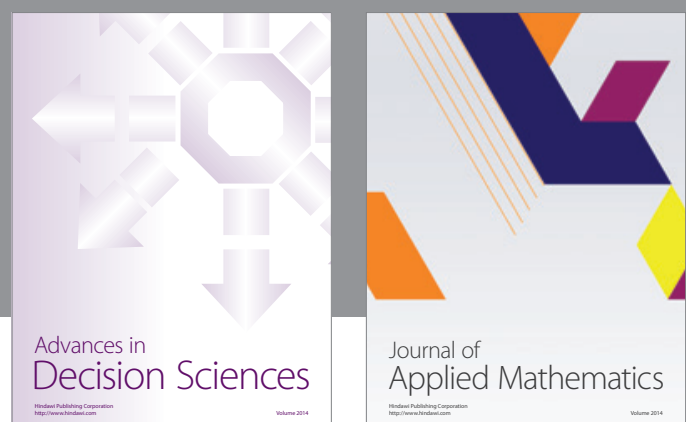

Journal of

Applied Mathematics
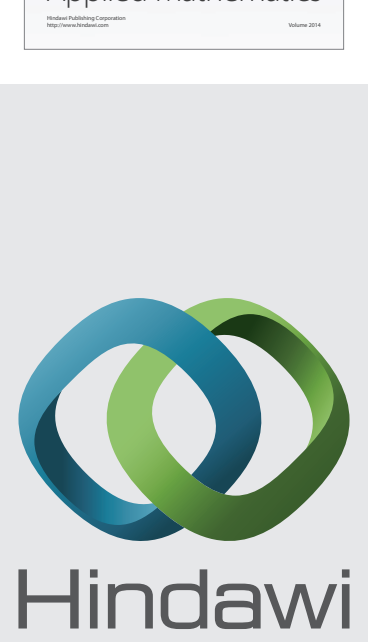

Submit your manuscripts at http://www.hindawi.com
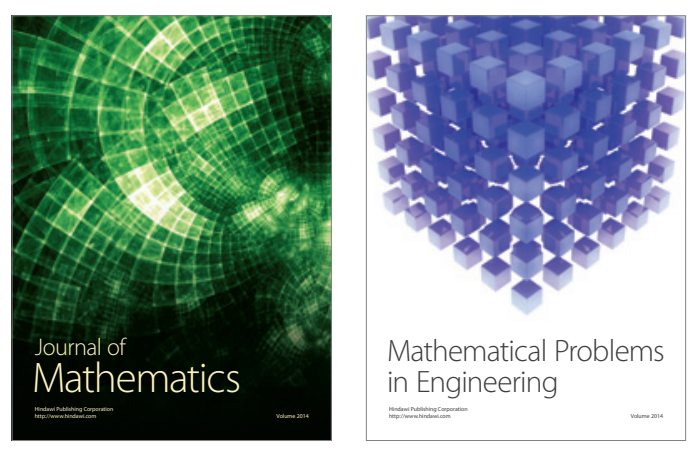

Mathematical Problems in Engineering
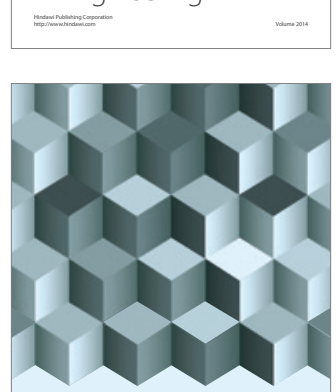

Journal of

Function Spaces
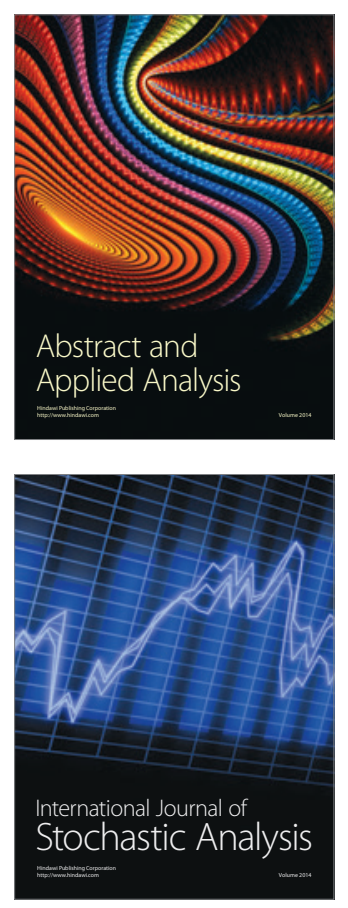

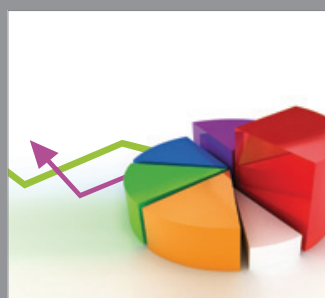

ournal of

Probability and Statistics

Promensencen
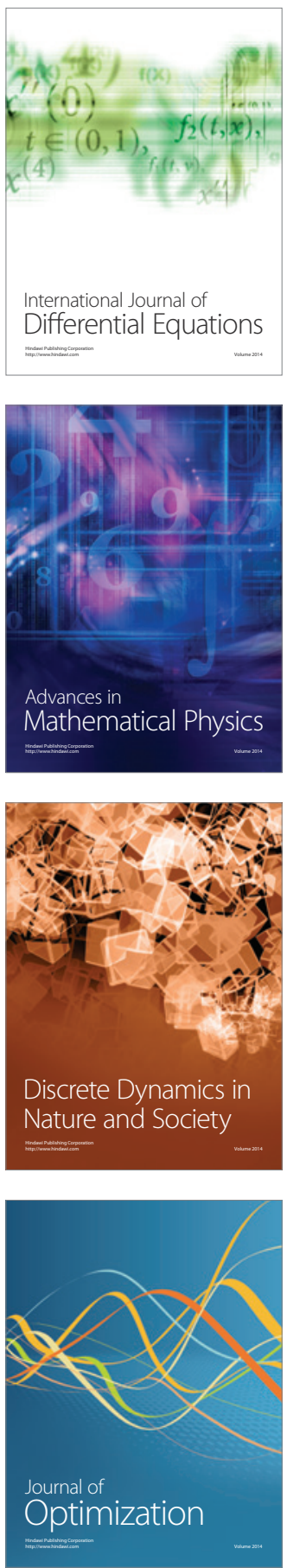